\title{
Open questions in Utility Theory
}

In honour of G.B. Mehta on occasion of his 75th birthday. Also dedicated to the memory of our colleague Gerhard Herden.

\author{
M.J. Campión ${ }^{1}$ and E. Induráin ${ }^{2}$
}

1 Inarbe (Institute for Advanced Research in Business and Economics) and Departamento de Estadística, Informática y Matemáticas. Universidad Pública de Navarra. 31005 Pamplona (SPAIN).e-mail: mjesus.campion@unavarra.es

2 InaMat (Institute for Advanced Materials) and Departamento de Estadística, Informática y Matemáticas. Universidad Pública de Navarra. 31005 Pamplona (SPAIN).e-mail: steiner@unavarra.es

\begin{abstract}
Throughout this paper, our main idea is to explore different classical questions arising in Utility Theory, with a particular attention to those that lean on numerical representations of preference orderings. We intend to present a survey of open questions in that discipline, also showing the state-of-art of the corresponding literature.
\end{abstract}

Keywords: Utility theory; ordered structures; numerical representability; existence of utility functions; special kinds of utility functions.

\section{Introduction}

Many researches in the topic of ordered structures and its numerical representations would undoubtedly agree on the fact that the book [17], by D.S. Bridges and G.B. Mehta, published in 1995, is one of the best treatises on this kind of question. In fact, that book has been a cornerstone, the bedside book of many professional colleagues. It covers from different perspectives and alternative points of view, the most important topics that are in the basis of the search for representations of preferences through utility functions.

Needless to say, not everything has been done. Classical problems, as, e.g., the existence of a numerical representation for a given semiorder, in the classical approach introcuced by Scott and Suppes (see [77, 38]), have been solved in the 
last ten years. But it is also true that some crucial questions still resist to the researchers, and remain as open.

Therefore, it seems necessary to dispose at hand of a commented account of open problems arising in utility theory, at least in all what has to do with the numerical representations of the main different -and classical- kinds of ordered structures, already considered in [17], namely total preorders, interval orders and semiorders.

This is the idea that inspires the present manuscript.

\section{Preliminaries}

\subsection{Basic definitions}

Henceforward $X$ will denote a nonempty set, and $\mathcal{R}$ will stand for a binary relation defined on $X$, that is $\mathcal{R}$ is a subset of $X^{2}$. Given $x, y \in X$, with the standard notation $x \mathcal{R} y$ we mean that $(x, y) \in \mathcal{R} \subseteq X \times X$.

Definition 2.1. A binary relation $\mathcal{R}$ on $X$ is called:

(i) Reflexive: If $x \mathcal{R} x$ holds for every $x \in X$.

(ii) Transitive: If $(x \mathcal{R} y) \wedge(y \mathcal{R} z) \Longrightarrow x \mathcal{R} z$ holds for every $x, y, z \in X$.

(iii) Total or complete: If $(x \mathcal{R} y) \vee(y \mathcal{R} x)$ holds for every $x, y \in X$.

(iv) Irreflexive: If for any $x \in X$, it never happens that $x \mathcal{R} x$.

(v) Asymmetric: If for any $x, y \in X$ such that $x \mathcal{R} y$ holds, it never happens that $y \mathcal{R} x$.

(vi) Antisymmetric: If $(x \mathcal{R} y) \wedge(y \mathcal{R} x) \Longrightarrow x=y$ holds for any $x, y \in X$.

(vii) Negatively transitive: If $x \mathcal{R} z \Longrightarrow(x \mathcal{R} y) \vee(y \mathcal{R} z)$ holds for all $x, y, z \in X$.

Associated to a binary relation $\mathcal{R}$ on a set $X$, we consider its negation (respectively, its transpose) as the binary relation $\mathcal{R}^{c}$ (respectively, $\mathcal{R}^{t}$ ) on $X$ given by $(x, y) \in \mathcal{R}^{c} \Leftrightarrow(x, y) \notin \mathcal{R}$ for every $x, y \in X$ (respectively, given by $(x, y) \in \mathcal{R}^{t} \Leftrightarrow(y, x) \in \mathcal{R}$, for every $\left.x, y \in X\right)$. We also define the adjoint $\mathcal{R}^{a}$ of the given relation $\mathcal{R}$, as $\mathcal{R}^{a}=\left(\mathcal{R}^{t}\right)^{c}$.

In the particular case in which some ordering has been implemented, the standard notation usually changes. We include it here for the sake of completeness, since we will use it throughout the present manuscript.

Definition 2.2. A preorder $\precsim$ on $X$ is a reflexive and transitive binary relation defined on $X$. An antisymmetric preorder is called an order, whereas a total preorder $\precsim$ is a preorder such that if $x, y \in X$ then $(x \precsim y) \vee(y \precsim x)$ holds. A total order is also known as a linear order in this literature.

If $\precsim$ is a preorder on $X$, then as usual we denote the associated asymmetric relation by $\prec$ and the associated equivalence relation by $\sim$ and these are defined 
by $x \prec y \Leftrightarrow(x \precsim y) \wedge \neg(y \precsim x)$ and $x \sim y \Leftrightarrow(x \precsim y) \wedge(y \precsim x)$. (Here the symbol " $\neg$ " stands for negation).

Remark 2.3. In the case of a total preorder $\precsim$, we may observe that $x \precsim y \Leftrightarrow$ $(x \prec y) \wedge(x \sim y)$ holds for all $x, y \in X$. Also, in this case the adjoint of $\precsim$ is $\prec$ and vice versa.

Definition 2.4. An interval order $\prec$ is an asymmetric binary relation on $X$ such that $(x \prec y) \wedge(z \prec t) \Rightarrow(x \prec t) \vee(z \prec y)(x, y, z, t \in X)$. Its corresponding adjoint will be denoted $\precsim$, so that $a \precsim b \Leftrightarrow \neg(b \prec a)$. This relation $\precsim$ is called the weak preference associated to $\prec$. By the way, $\prec$ is also called a strict preference defined on $X$. In addition, the binary relation $\sim$ defined by $a \sim b \Leftrightarrow(a \precsim b) \wedge(b \precsim a)$ is said to be the indifference associated to $\prec$.

Remark 2.5. It is well known that given an interval order $\prec$ on a set $X$, the associated relations $\precsim$ and $\sim$ may fail to be transitive $([56,57,58,66,77])$.

Definition 2.6. An interval order $\prec$ is said to be a semiorder if $(x \prec y) \wedge$ $(y \prec z) \Rightarrow(x \prec w) \vee(w \prec z)(x, y, z, w \in X)$. A semiorder $\prec$ is said to be typical if its associated weak preference $\precsim$ defined by $x \precsim y \Leftrightarrow \neg(y \prec x),(x, y \in X)$ is not a total preorder on $X$.

\subsection{Numerical representations of different kinds of order- ings}

Following [9] we introduce the notion of representability for different kinds of orderings.

The underlying idea corresponds to the possibility of converting qualitative scales into quantitative ones. Thus, in a way, a binary relation on a nonempty set $X$ could give us the idea of comparison, preference, choice, better position, etc., depending on the context. For instance, given $a, b \in X$, the fact $a \precsim b$ could be interpreted as " $b$ is at least as good as $a$ ". Obviously, it is more practice to compare directly real numbers rather than dealing with qualitative scales. This suggest us to convert, when possible, a qualitative scale into a quantitative or numerical one.

Definition 2.7. A total preorder $\precsim$ on $X$ is called representable if there is a real-valued function $u: X \rightarrow \mathbb{R}$ that is order-preserving, so that, for every $x, y \in X$, it holds that $x \precsim y \Leftrightarrow u(x) \leq u(y)$. The map $u$ is said to be a utility function for $\precsim^{1}$.

An interval order $\prec$ defined on $X$ is said to be representable (as an interval order) if there exist two real valued maps $u, v: X \longrightarrow \mathbb{R}$ such that $x \prec y \Leftrightarrow$ $v(x)<u(y) \quad(x, y \in X)$. The pair $(u, v)$ is called a utility pair representing $\prec$.

A semiorder $\prec$ defined on $X$ is said to be representable in the sense of Scott and Suppes if there exists a real-valued map $u: X \rightarrow \mathbb{R}$ (again called a utility

\footnotetext{
${ }^{1}$ In other quite different and multidisciplinary contexts, this is sometimes called an isotony, an entropy function, a measurement, a score or an order-preserving map. (See e.g. [8, 10, 17, 18, 30, 31, 43, 49, 77])
} 
function) such that $x \prec y \Leftrightarrow u(x)+1<u(y)(x, y \in X)$ (see the seminal reference [77]).

In this case, the pair $(u, 1)$ is said to be a Scott-Suppes representation of $\prec$.

Remark 2.8. If $(u, v)$ is a utility pair representing an interval order $\prec$ defined on a set $X$, it is straightforward to see that $u(x) \leq v(x)$ for every $x \in X$. The real interval $[u(x), v(x)]$, that could degenerate to a point if $u(x)=v(x)$, is said to be the interval of discrimination or perception corresponding to the element $x \in X$. And the non-negative real number $v(x)-u(x)$ is said to be the discrimination threshold for the element $x \in X$. Notice that these thresholds depend on the elements of $X$. If $x \neq y \in X$ it may happen that $v(x)-u(x) \neq$ $v(y)-u(y)$. In the case of a semiorder that is representable in the sense of Scott and Suppes, the discrimination thresholds are all equal to 1.

There exist interval orders that fail to be representable (as interval orders). Also, there exist semiorders that are not representable in the sense of Scott and Suppes. By the way, since every semiorder is, by definition, a special case of an interval order, it may still happen that some particular semiorder can be represented as an interval order through a utility pair, but it fails to be representable as a semiorder in the sense of Scott and Suppes (see [41, 73] for suitable examples and further information).

Definition 2.9. Associated to an interval order $\prec$ defined on a nonempty set $X$, we shall consider three new binary relations (see $[4,48,55,56]$ ).

These binary relations are said to be the traces of $\prec$. They are respectively denoted by $\prec^{*}$ (left trace), $\prec^{* *}$ (right trace) and $\prec^{0}$ (main trace), and defined as follows: $x \prec^{*} y \Leftrightarrow x \prec z \precsim y$ for some $z \in X$, and similarly $x \prec^{* *}$ $y \Leftrightarrow x \precsim z \prec y \quad$ for some $z \in X \quad(x, y \in X)$. In addition, $x \prec^{0} y \Leftrightarrow\left(x \prec \prec^{*}\right.$ $y) \vee\left(x \prec \prec^{* *} y\right) \quad(x, y \in X)$.

Remark 2.10. We denote $x \precsim^{*} y \Leftrightarrow \neg\left(y \prec^{*} x\right), \quad x \sim^{*} y \Leftrightarrow x \precsim \precsim^{*} y \precsim^{*} x$, $x \precsim^{* *} y \Leftrightarrow \neg\left(y \prec^{* *} x\right)$ and $x \sim^{* *} y \Leftrightarrow x \precsim^{* *} y \precsim^{* *} x$, and finally $x \precsim^{0}$ $y \Leftrightarrow\left(x \precsim^{*} y\right) \wedge\left(x \precsim^{* *} y\right)$ and $x \sim^{0} y \Leftrightarrow\left(x \precsim^{0} y\right) \wedge\left(y \precsim^{0} x\right) \quad(x, y \in \widetilde{X})$. Both the binary relations $\precsim^{*}$ and $\precsim^{* *}$ are total preorders on $X$. Moreover, the indifference relation $\sim$ associated to the interval order $\prec$ is transitive if and only if $\precsim^{*}, \precsim^{* *}$ and $\precsim$ coincide. In this case $\precsim$ is actually a total preorder on $X$ (see $[12,48,55,56,70,73])$.

In addition, the binary relation $\precsim^{0}$ allows us to characterize semiorders among interval orders (see $[55,56,70]$ ).

Indeed, if $\prec$ an interval order on $X$, then it is a semiorder if and only if $\precsim^{0}$ is a total preorder on $X$. A semiorder $\prec$ is not typical if and only if $\precsim^{*}, \precsim^{* *}, \precsim^{0}$ and $\precsim$ coincide.

Let us recall now some characterizations of the numerical representability of total preorders, interval orders and semiorders.

Definition 2.11. Let $X$ be a nonempty set. A total preorder $\precsim$ defined on $X$ is said to be perfectly separable if there exists a countable subset $D \subseteq X$ such that for every $x, y \in X$ with $x \prec y$ there exists $d \in D$ such that $x \precsim d \precsim y$. 
An interval order $\prec$ defined on $X$ is said to be interval order separable if there exists a countable subset $D \subseteq X$ such that for every $x, y \in X$ with $x \prec y$ there exists $d \in D$ such that $x \precsim^{*} d \prec y$. Equivalently, for every $x, y \in X$ with $x \prec y$ there exists $c \in D$ such that $x \prec c \precsim{ }^{* *} y$ (see $[9,12]$ ).

A semiorder $\prec$ defined on $X$ is said to be regular with respect to sequences if for any $x, y \in X$, and sequences $\left(x_{n}\right)_{n \in \mathbb{N}},\left(y_{n}\right)_{n \in \mathbb{N}} \subseteq X$, none of the situations $x \prec \cdots \prec x_{n+1} \prec x_{n} \prec \cdots \prec x_{1}$ and $y_{1} \prec \cdots \prec y_{n} \prec y_{n+1} \prec \cdots \prec y$ may occur.

The following facts are well-known in this literature (see $[12,17,73]$ ).

Theorem 2.12. On a nonempty set $X$ the following statements hold:

(a) A total preorder $\precsim$ is representable if and only if it is perfectly separable.

(b) An interval order $\prec$ is representable if and only if it is interval order separable.

And the following result has recently been proved (see [35, 38]).

Theorem 2.13. Let $X$ be a nonempty set. Let $\prec$ be a typical semiorder defined on $X$. Then, $\prec$ is representable in the sense of Scott and Suppes if and only if it is both interval order separable and regular with respect to sequences.

Remark 2.14. When $X$ is countable, the condition of interval order separability trivially holds. Therefore, a semiorder $\prec$ on a countable set $X$ is representable in the sense of Scott and Suppes if and only if it is regular with respect to sequences. This fact was already known $([7,68])$.

\subsection{Order topology}

Definition 2.15. Let $\prec$ denote an asymmetric binary relation on $X$. Given $a \in X$ the sets $L(a)=\{t \in X: t \prec a\}$ and $U(a)=\{t \in X: a \prec t\}$ are called, respectively, the lower and upper contours of $a$ relative to $\prec$. We say that $\prec$ is $\tau$-continuous if for each $a \in X$ the sets $L(a)$ and $U(a)$ are $\tau$-open. (See also [64] for further details). The minimal topology $\tau_{\prec}$ for which all the contours $L(a)$ and $U(a)$ are open $(a \in X)$ is said to be the topology generated for $\prec$. When $\prec$ is the asymmetric part of a total preorder $\precsim$, the topology $\tau_{\prec}$ is also known as the order topology associated to $\precsim$. Sometimes it is also denoted by $\tau \precsim$. Notice that a subbasis for this topology is $\{\emptyset\} \cup\{X\} \bigcup\{U(a): a \in X\} \bigcup\{L(\widetilde{a}): a \in X\}$.

If $\precsim$ is a total preorder on $X$, a topology $\tau$ on $X$ is said to be natural as regards $\precsim$ if $\prec$ is $\tau$-continuous, or equivalently, if the topology $\tau \prec$ is coarser than $\tau$ (i.e., $\tau$ is finer than $\tau_{\prec}$. We also say that $\tau_{\prec}$ is a subtopology of $\tau$ ).

The order topology $\tau_{\prec}$ associated to a total preorder $\precsim$ defined on a nonempty set $X$ characterizes in topological terms the numerical representability of $\precsim$ through a utility function, as follows:

Theorem 2.16. A total preorder $\precsim$ on a nonempty set $X$ is representable if and only if its associated order topology $\tau_{\prec}$ is second countable.

Proof. See Sections 1.4 and 1.6 in [17]. 


\subsection{Debreu's open gap lemma and the continuous repre- sentability of total preorders}

Debreu's open gap lemma is a powerful tool to obtain continuous representations of a total preorder $\precsim$ defined on a nonempty set $X$ endowed with a natural topology $\tau$. (See Ch. 3 in [17], as well as [25] and [46]). To explain this lemma, we introduce the following definition.

Definition 2.17. Let $S$ be a subset of the real line $\mathbb{R}$. A lacuna $L$ corresponding to $S$ is a non degenerate interval of $\mathbb{R}$ that has both a lower bound and an upper bound in $S$ and that has no points in common with $S$. A maximal lacuna is said to be a Debreu gap (see e.g. pp. 38 and ff. in [17]).

Lemma 2.18. If $S$ is a subset of the extended real line $\overline{\mathbb{R}}$, then there exists a strictly increasing map, called a gap function, $g: S \longrightarrow \overline{\mathbb{R}}$ such that all the Debreu gaps of $g(S)$ are open.

Proof. See Section 3.1 in [17].

Theorem 2.19. A total preorder $\precsim$ on a topological space $(X, \tau)$ is representable through a continuous utility function if and only if $\precsim$ is perfectly separable and the topology $\tau$ is natural for $\precsim$.

Proof. See Theorem 3.2.9 in [17]. We outline here the main ideas of the proof of this result, for the sake of completeness. Using Debreu's open gap lemma, the classical method to get a continuous real-valued strictly isotone function goes as follows: First, one constructs a strictly isotone function (which may not necessarily be continuous) $f$, whose existence is guaranteed by part (a) of Theorem 2.12 above (for a further account, see e.g. [8], Theorem 24 on p. 200, or else [17], Theorem 1.4.8 on p. 14). Then Debreu's open gap lemma is applied to find a strictly increasing function $g: f(X) \longrightarrow \mathbb{R}$ such that all the Debreu gaps of $g(f(X))$ are open. Consequently, the composition $F=g \circ f: X \longrightarrow \mathbb{R}$ is also a utility representation for the preorder $\precsim$ defined on $X$, but now $F$ is continuous with respect to any given natural topology $\tau$ on $X$.

From Theorem 2.19 and Theorem 2.12, next Corollary 2.20 is easily achieved.

Corollary 2.20. Let $(X, \tau)$ be a topological space. Suppose that $X$ is endowed with a representable total preorder $\precsim$. Assume also that the topology $\tau$ is natural for . Then $\precsim$ is continuously representable.

\subsection{The problem of the continuous representability of in- terval orders and semiorders}

Unlike the case of total preorders, no general characterization of the continuous representability of interval orders and semiorders has been achieved yet.

At this stage one may expect some result similar to Theorem 2.19, maybe substituting perfectly separable by interval order separable or perhaps by interval order separable plus regular with respect to sequences. Moreover, we would 
also need to introduce a concept as natural topology with respect to an interval order or to a semiorder. But, unfortunately, these attempts do not work in general, as proved in [9].

Since in Theorem 2.12 the condition of interval order separability is crucial, and that condition involves the traces, in order to analyze the continuous representability of interval orders it seems reasonable to introduce the following definition, which adapts to interval orders the notion of a natural topology, formerly introduced for total preorders.

Definition 2.21. Let $X$ be a nonempty set endowed with a topology $\tau$. Let $\prec$ be an interval order on $X$. We say that $\tau$ is natural as regards $\prec$, if all the upper and lower contour sets of $\prec, \prec{ }^{*}$ and $\prec^{* *}$ are $\tau$-open.

The main result in [9], namely Theorem 2.22 below, gives a characterization of the continuous representability of an interval order provided that some additional conditions hold. That is, it does not constitute yet a general characterization of the continuous representability of an interval order. However, up-to-date it is, perhaps, the best achievement in this direction.

Theorem 2.22. Let $\prec$ denote an interval order defined on a nonempty set $X$ endowed with a topology $\tau$. There exists a pair of continuous functions $f, g$ : $X \rightarrow \mathbb{R}$, where on $X$ we consider the topology $\tau$, and $\mathbb{R}$ is endowed with the usual Euclidean one, such that the pair $(f, g)$ represents $\prec$ and in addition $f$ is a representation for the associated trace ${ }^{* *}$ (which is a total preorder) and $g$ is a representation for the total preorder $\precsim^{*}$ if and only $\prec$ is interval order separable and $\tau$ is a natural topology with respect to $\prec$.

Proof. See Theorem 1 in [9].

Remark 2.23. Notice that we may have continuous representations $(f, g)$ of an interval order $\prec$ such that $f$ does not represent $\precsim^{* *}$ or $g$ fails to represent $\precsim *$. Furthermore, unlike the situation for total preorders, if an interval order $\prec$ on a topological space $(X, \tau)$ admits a continuous representation, the topology $\tau$ could still fail to be natural as regards $\prec$. (See $[9,11]$ for further details).

Other (partial) characterizations of the continuous representability of interval orders provided that some topological condition, different from the fact of the topology being natural with respect to the interval order, is accomplished a priori have been achieved in [13]. Among them, it is noticeable that a full characterization of the continuous representability of an interval order has been obtained provided that the set $X$ is finite. (See subsection 4.2 in [11]).

Following [11], where the results that follow below in this subsection 2.5 were already proved, we furnish now several necessary conditions for the representability of an interval order through a pair of continuous real-valued functions, in the general case.

Lemma 2.24. Let $\prec$ denote an interval order defined on a nonempty set $X$. Assume that $\prec$ is representable by means of a pair $(u, v)$ of real-valued functions. Then, the following statements hold for any $x, y \in X$ : 
(i) $x \prec^{*} y \Longrightarrow v(x)<v(y)$,

(ii) $x \prec^{* *} y \Longrightarrow u(x)<u(y)$,

(iii) $v(x)=v(y) \Longrightarrow x \sim^{*} y$,

(iv) $u(x)=u(y) \Longrightarrow x \sim^{* *} y$.

Proposition 2.25. Let $(X, \tau)$ be a topological space endowed with an interval order $\prec$. If the interval order is representable through a pair $(u, v)$ of continuous real-valued functions, then the following properties hold:

(i) The interval order is $\tau$-continuous.

(ii) If a net $\left(x_{j}\right)_{j \in J} \subseteq X$ converges to two points $a, b \in X$, then $a \sim^{0} b$.

(iii) If a net $\left(x_{j}\right)_{j \in J} \subseteq X$ converges to $a \in X$, and $b, c \in X$ are such that $x_{j} \prec b \precsim a$ and $x_{j} \prec c \precsim a$ for any $j \in J$, then $b \sim^{* *} c$.

(iv) If a net $\left(x_{j}\right)_{j \in J} \subseteq X$ converges to $a \in X$, and $b, c \in X$ are such that $a \precsim b \prec x_{j}$ and $a \precsim c \prec x_{j}$ for any $j \in J$, then $b \sim^{*} c$.

The characterization of the continuous representability of interval orders on a topological space $(X, \tau)$ of finite support goes as follows:

Theorem 2.26. Let $(X, \tau)$ be a topological space in which the set $X$ is finite. Let $\prec$ be an interval order defined on $X$. The following statements are all equivalent:

(i) The interval order $\prec$ has a representation by means of a pair $(u, v)$ of continuous real-valued functions.

(ii) The interval order $\prec$ satisfies the necessary conditions (i)-(iv) introduced in Proposition 2.25.

(iii) The interval order $\prec$ satisfies the condition (ii) introduced in Proposition 2.25, namely, if a net $\left(x_{j}\right)_{j \in J} \subseteq X$ converges to two points $a, b \in X$, then $a \sim^{0} b$.

(iv) The topology $\tau$ is natural with respect to the interval order $\prec$.

The situation for semiorders, looking for continuous Scott-Suppes representations is even more deceiving. Only a few partial results are known (see $[28,53])$. Among them, one of the best achievements is the characterization of the continuous representability of semiorders on a topological space $(X, \tau)$ in which the set $X$ is finite.

Theorem 2.27. Let $(X, \tau)$ stand for a topological space in which the set $X$ is finite. Let $\prec$ be a semiorder on $X$. Then $\prec$ admits a continuous Scott-Suppes representation if and only if for any net $\left(x_{j}\right)_{j \in J}$ in $X$ it happens that if the net converges to two points $a, b \in X$ then $a \sim^{0} b$ holds. 
Proof. See Section 4 in [53].

Matching Theorem 2.26 and Theorem 2.27, the following straightforward corollary arises.

Corollary 2.28. Let $(X, \tau)$ be a topological space in which the set $X$ is finite. Let $\prec$ be a semiorder defined on $X$. The following statements are equivalent:

(i) The binary relation $\prec$ admits a continuous representation as an interval order, through a pair $(f, g)$ of continuous real-valued functions $f, g: X \rightarrow$ $\mathbb{R}$ such that $a \prec b \Leftrightarrow g(a)<f(b)$ holds for every $a, b \in X$.

(ii) The binary relation $\prec$ admits a continuous representation as a semiorder, by means of a continuous function $u: X \rightarrow \mathbb{R}$ such that $a \prec b \Leftrightarrow u(a)+1<$ $u(b)$ holds for every $a, b \in X$.

\section{Open questions related to numerical repre- sentability}

In this section we start with the task of furnishing the reader an explicit list of open questions related to utility theory, paying a particular attention to the numerical representability (without additional conditions such as, e.g., continuity), passing then to give an account of open questions concerning the continuous representability of several kinds of ordered structures on a topological space.

\subsection{Open problems about numerical representability of bi- nary relations}

In general, most of the open questions relative to the numerical representations of binary relations appear when dealing with additional structures, in particular the topological (e.g.: concerning continuity) and the algebraic ones.

However, there are still some open questions that appear in this setting, without any additional structure on a given nonempty set $X$, but related to the numerical representation of some binary relation $\mathcal{R}$ (that is, a qualitative scale on $X$ ), provided that it satisfies some natural restriction (e.g.: acyclicity).

Definition 3.1. A binary relation $\mathcal{R}$ on a nonempty set $X$ is said to be acyclic if for any $\left(x_{1}, \ldots, x_{n}\right) \in X^{n}$ such that $x_{i} \mathcal{R} x_{i+1}(i=1, \ldots, n-1)$ it holds that $\left(x_{n}, x_{1}\right) \notin \mathcal{R}$ (in other words, $x_{n} \mathcal{R} x_{1}$ cannot hold).

Problem 3.2. It is an open question to find some kind of numerical representation that could convert and (re)-interprete an acyclic binary relation (as a qualitative scale) into one or more numerical or quantitative scales. Some partial results in this direction were issued in [3].

Problem 3.3. Other open questions follow just the opposite order of ideas in Problem 3.2. That is, suppose that we have one or more numerical scales 
of a certain type, and we want to identify exactly which type of binary relation they represent. To put just an example, bearing in mind the definition of a representable interval order, suppose that we are given three realvalued functions $f, g, h: X \longrightarrow \mathbb{R}$, where $X$ is a nonempty set. We wonder which are the main abstract properties of the binary relation $\mathcal{R}$ defined by $x \mathcal{R} y \Leftrightarrow f(x)<g(y)<h(x) \quad(x, y \in X)$. Conversely, given a binary relation $\mathcal{R}$ on $X$ we may ask ourselves if there exists a trio of real-valued functions $f, g, h: X \longrightarrow \mathbb{R}$ such that $x \mathcal{R} y \Leftrightarrow f(x)<g(y)<h(x) \quad(x, y \in X)$ holds.

Some results in a close direction appeared in [79], but, in general, problems of this kind remain still open.

Another interesting source of open problems comes to our mind when we analyze non-representability. To fix our ideas, suppose that we are studying the numerical representability of total preorders through a real-valued utility function. Due to the characterization stated in Theorem 2.12, one may give examples of non-representable total preorders: consider for instance the plane $\mathbb{R}^{2}$ endowed with the lexicographic ordering $\precsim L$ given by $(a, b) \precsim L(c, d) \Leftrightarrow(a<$ $c) \vee[(a=c) \wedge(b \leq d)] \quad(a, b, c, d \in \mathbb{R})$ (see e.g. pp. 10 and ff. in [17] for further details). At this point we may wonder if there some minimal non-representable total preorder (up-to-isomorphisms) such that any non-representable total preorder should contain an isotonic copy of it. Those minimal non-representable totally preordered structures (if there is any) would then act as germs of nonrepresentability. Each time that a total preorder contains a copy of one of those germs, we could immediately say that it fails to be representable.

For the case of total orders this problem was studied in depth in [6], and the corresponding main germs of non-representability were identified. These are the lexicographic plane, as well as, on the one hand, the so-called long line and on the other hand the Aronszajn chains (see [6] for definitions and further details).

\section{Problem 3.4.}

(i) Analyze the existence of germs of non-representability for interval orders.

(ii) Analyze the existence of germs of non-representability (in the sense of Scott and Suppes) for semiorders.

\subsection{Continuous utility functions that represent different kinds of orderings: the state-of-art}

The study of the existence of continuous representations of different kinds of orderings is a substantial part of the analysis of the interaction between Order and Topology. It is important to realize that when we have a nonempty set $X$ endowed with a a topology $\tau$ and some ordering of a certain class (e.g.: a total preorder $\precsim$ ), the topological properties of $\tau$ may force the ordering to have some special features, and vice versa. A pioneer work on this interaction was due to Leopoldo Nachbin in the 1950's but published as a book much later (see [71]), and nowadays it is a classical reference in this literature. Conceptually, it is quite 
important to realize that having at hand a set $X$ endowed with two structures of different kind (namely, its topology and ordering) may give rise to new situations in which it could not be enough to study those structures (topology vs. order) independently, as watertight compartments or pigeon holes.

In addition, the mere existence of a continuous representation for a binary relation $\mathcal{R}$ defined on a topological space $(X, \tau)$ may also induce on both $\tau$ and $\mathcal{R}$ some restrictive additional properties. To put an example, and as a straightforward exercise, consider that $\mathcal{R}$ has a numerical representation through a real-valued function $F: X \rightarrow \mathbb{R}$ such that $a \mathcal{R} b \Leftrightarrow F(a) \leq F(b)$ holds for every $a, b \in X$ and, additionally, $F$ is continuous with respect to the topology $\tau$ in $X$ and the usual Euclidean topology on the real line. Then, it easily follows that $\mathcal{R}$ is a total preorder on $X$ that is actually $\tau$-continuous. (In other words, the topology $\tau$ is natural as regards the total preorder $\mathcal{R}$ ). Needless to say, when two or more functions are involved in the numerical representation (as, e.g., when representing interval orders), the situation could be much more subtle and feature many more nuances.

Among the classical orderings that may be defined on a nonempty set $X$, namely total preorders, interval orders and semiorders, the continuous representability has only been characterized for the case of total preorders (Theorem 2.19 above). In the case of interval orders and semiorders, it has only been characterized for the case in which $X$ is finite (Theorem 2.26 and Theorem 2.27 above). Also, for the continuous representability of interval orders, other partial characterizations known use some additional restrictions in their corresponding statements (see e.g. Theorem 2.22 above). They are not valid for the general case.

For other binary relations $\mathcal{R}$ on a topological space $(X, \tau)$ the general situation about the possibility of converting the qualitative scale $\mathbb{R}$ into one of more quantitative or numerical ones, by means some ad hoc kind of continuous functions constitutes an open framework of research in this literature.

Problem 3.5. Given a topological space $(X, \tau)$ and a binary relation $\mathcal{R}$ defined on it, of particular features, analyze the existence of one or more continuous realvalued functions whose domain is $X$ such that they fully represent $\prec$ someway.

\subsection{Continuous numerical representability of interval or- ders and semiorders}

As aforesaid in the subsection 2.5 above, it is still an open problem to find a full characterization of the continuous representability of interval orders and semiorders. Only partial results have been got up-to-date.

Moreover, many collateral open questions appear as a by-product when studying those main questions. Consider the following example:

Problem 3.6. Which could be a suitable definition of a natural topology in the case of semiorders? Should we take into account the trace $\precsim^{0}$ ?

We explore these questions now, in order to furnish a further account of open problems in this setting. 


\section{Problem 3.7.}

(i) Given a topological space $(X, \tau)$ endowed with an interval order $\prec$, characterize the existence of a pair of real-valued functions $f, g: X \rightarrow \mathbb{R}$ that are continuous as regards the topology $\tau$ on $X$ and the usual one on the real line, and represent $\prec$ so that $x \prec y \Leftrightarrow g(x)<f(y)$ holds for every $x, y \in X$.

(ii) Given a topological space $(X, \tau)$ endowed with a semiorder $\prec$, characterize the existence of a real-valued utility function $u: X \rightarrow \mathbb{R}$ that is continuous as regards the topology $\tau$ on $X$ and the usual one on the real line, and represents $\prec$ in the sense of Scott and Suppes, so that $x \prec y \Leftrightarrow u(x)+1<$ $u(y)$ holds for every $x, y \in X$.

(iii) Given a topological space $(X, \tau)$ endowed with a semiorder $\prec$, characterize the existence of a pair of real-valued functions $f, g: X \rightarrow \mathbb{R}$ that are continuous as regards the topology $\tau$ on $X$ and the usual one on the real line, and represent $\prec$ so that $x \prec y \Leftrightarrow g(x)<f(y)$ holds for every $x, y \in X$. (In other words, the semiorder $\prec$, considered just as an interval order, is continuously representable).

(iv) Characterize the topological spaces $(X, \tau)$ for which a semiorder $\prec$ that is representable in the sense of Scott and Suppes actually admits a continuous Scott-Suppes representation. (In other words, study the topological spaces $(X, \prec)$ for which a generalization of Corollary 2.20 to semiorders and Scott-Suppes representations is still possible).

(v) Given a topological space $(X, \tau)$ and a semiorder $\prec$ that is representable as an interval order through a pair of real-valued functions $f, g: X \rightarrow \mathbb{R}$ that are continuous as regards the topology $\tau$ on $X$ and the usual one on the real line, try to find a continuous and strictly increasing function $h: f(X) \cup g(X) \subseteq \mathbb{R} \longrightarrow \mathbb{R}$ such that $h(g(x))=h(f(x))+1$ holds for every $x \in X$.

Remark 3.8. Part (v) of Problem 3.7 is closely related to the study of a classical functional equation on a single real variable, known in the literature as the Abel's functional equation (see [1] for further details). Notice here that when the function $h$ exists, we get a continuous Scott-Suppes representation of the semiorder $\prec$ since, calling $u=h \circ f$ we have that $x \prec y \Leftrightarrow g(x)<f(y) \Leftrightarrow$ $h(g(x))<h(f(y)) \Leftrightarrow h(f(x))+1<h(g(y)) \Leftrightarrow u(x)+1<u(y) \quad(x, y \in X)$.

\section{The continuous representability properties in General Topology}

Given a nonempty set $X$, in this section we study topologies through the properties that they induce on different kinds of orderings that may be defined on the given set $X$. 


\subsection{The continuous representability property (CRP)}

Following $[20,23,24,26]$ we introduce the following key definition. (The corresponding concept had also been analyzed in [60,62], but under the name of a useful topology).

Definition 4.1. Let $(X, \tau)$ be a topological space. We say that the topology $\tau$ satisfies the continuous representability property (CRP) if every $\tau$-continuous total preorder $\precsim$ defined on $X$ admits a continuous utility representation by means of an order-preserving real-valued function $u: X \longrightarrow \mathbb{R}$ such that $x \precsim$ $y \Leftrightarrow u(x) \leq u(y)$ holds for every $x, y \in X$, and $u$ is continuous with respect to the topology tau on $X$ and the usual topology on $\mathbb{R}$.

Definition 4.2. Given a topological space $(X, \tau)$ we say that the topology $\tau$ is preorderable (respectively, orderable) if it coincides with the order topology $\tau_{\prec}$ of some total preorder (respectively, total order) $\precsim$ defined on $X$.

Remark 4.3. The orderability of a topology was studied and characterized in $[74,80]$ in terms of the existence of subbases accomplishing some list of suitable properties. Completing the panorama, the preorderability of a topology was also characterized in Theorem 3.1 (ii) in [24].

Problem 4.4. As mentioned above, topological spaces $(X, \tau)$ such that the topology $\tau$ is the order topology of some total preorder on $X$ were characterized in [24]. But nothing similar is known for topologies induced by other different kinds of binary relations (not necessarily total preorders), see e.g. [64]. Thus, given an asymmetric binary relation $\mathcal{R}$ on a nonempty set $X$, we can also consider the sets of the form $U(x)=\{a \in X: x \mathcal{R} a\}(x \in X)$ as well as the sets $L(x)=\{b \in X: b \mathcal{R} x\}(x \in X)$, and consequently define a topology $\tau_{\mathcal{R}}$ on $X$, a subbasis of which is given by $\{\emptyset\} \cup\{X\} \bigcup\{U(t): t \in X\} \bigcup\{L(y): y \in X\}$.

Here it is no longer true, in general, that the binary relation $\mathcal{R}^{a}$, adjoint of $\mathcal{R}$, is a total preorder. Important particular cases correspond to interval orders and semiorders. In this framework, it is an open problem to characterize topologies that coincide with the topology $\tau_{\prec}$ induced by an interval order or a semiorder $\prec$ defined on the given set $X$.

The property CRP has also been characterized in topological terms as follows:

Theorem 4.5. Let $(X, \tau)$ be a topological space. The topology $\tau$ satisfies the continuous representability property if and only if every topology $\tau^{\prime}$ on $X$ such that $\tau^{\prime}$ is coarser than $\tau$ (it is also said that $\tau^{\prime}$ is a subtopology of $\tau$ ), and preorderable, satisfies the second countability axiom.

Proof. See Theorem 5.1 in [24].

Problem 4.6. We could also say that a topological space $(X, \tau)$ satisfies the continuous representability property for interval orders (respectively, continuous representability property for semiorders) (CRP-I.O) (respectively (CRP-S) if for 
every interval order $\prec$ (respectively, for every semiorder) defined on $X$ and such that all the sets $U(x)=\{a \in X: x \mathcal{R} a\}(x \in X)$ and $L(x)=\{b \in X: b \mathcal{R} x\}(x \in$ $X)$ are $\tau$-open, there exists a continuous representation as an interval order (respectively, as a semiorder, in the sense of Scott and Suppes). The analysis and characterization of topological spaces that satisfy CRP-I.O or CRP-S is still an open problem.

Definition 4.7. A topological space $(X, \tau)$ is said to have the hereditary continuous representation property (HCRP) if every nonempty subset $Y \subseteq X$ has the continuous representation property $(\mathrm{CRP})$ with respect to the topology $\tau_{Y}$ that $\tau$ induces on $Y$.

Remark 4.8. It is obvious that HCRP implies CRP. But the converse is not true, as proved in Example 5.4 in [22] or Example 4.1 in [26].

Problem 4.9. It is an open question to characterize the topological spaces $(X, \tau)$ that satisfy the hereditary continuous representability property (HCRP).

Remark 4.10. Concerning classical topological properties encountered in General Topology, and its relationship with the continuous representability property (CRP), it is well-known that connected plus separable topological spaces satisfy CRP (see [51]). In addition, in [26] is has been proved that locally connected plus separable topological spaces also satisfy the continuous representability property. However, the converses are not true. See also Theorem 4.3 in [27] for additional information.

Problem 4.11. Are there other topological conditions (perhaps more general than separability, connectedness or local connectedness) that imply the continuous representability presentability property?

\subsection{The semicontinuous representability property (SRP)}

If we pay attention now to semicontinuity instead of continuity, some remarkable subtleties appear. Notice that given a topological space $(X, \tau)$ and a total preorder $\precsim$ on $X$ it could still happen that $\precsim$ admits a semicontinuous utility representation and $\tau$ fails to be a natural topology for $\precsim$. Indeed, if we pay attention to Theorem 2.19, we may observe that in order to pass from a mere utility representation of $\precsim$ to a continuous one, we needed $\tau$ to be a natural topology. But this could not be the case now. So, the following problem appears:

Problem 4.12. Let $(X, \tau)$ a topological space. Let $\precsim$ be a total preorder defined on $X$. Assume that the topology $\tau$ is not natural with respect to $\precsim$. Is it still possible to find a semicontinuous utility representation for $\precsim$ ?

Remark 4.13. An important result in this direction was obtained by T. Rader in 1963 (see $[69,75]$ ). Namely, Rader's utility representation theorem guarantees the existence of an upper semicontinuous utility function for any upper semicontinuous total preorder on a second countable topological space. 
Let us introduce now some helpful definitions, related to that question.

Definition 4.14. Let $(X, \tau)$ be a topological space. A real-valued function $f: X \longrightarrow \mathbb{R}$ is said to be lower (respectively upper) semicontinuous with respect to the topology $\tau$ on $X$ and the usual topology on $\mathbb{R}$, if for every $a \in \mathbb{R}$ the set $\{x \in X: a<f(x)\}$ (respectively, the set $\{x \in X: f(x)<a\}$ ) is $\tau$-open.

Definition 4.15. Let $(X, \tau)$ a topological space. Let $\precsim$ be a total preorder defined on $X$. Then $\precsim$ is said to be $\tau$-lower (respectively, $\tau$-upper) semicontinuous on $X$ if for each $x \in X$ the set $U(x)=\{y \in X: x \prec y\}$ (respectively, the set $L(x)=\{z \in X: z \prec x\}$ is $\tau$-open. The topology $\tau$ on $\mathrm{X}$ is said to have the semicontinuous representability property (SRP) if every semicontinuous total preorder defined on $X$ admits a representation by means of a semicontinuous order-monomorphism (of the same type of semicontinuity). Topologies satisfying SRP were studied in [15] under a different nomenclature. Then they have been analyzed in $[22,23,24,27]$.

Definition 4.16. Let $X$ be a nonempty set. Let $\precsim$ be a total preorder on $X$. The topology $\tau_{\prec}^{l}$ on $X$, a subbasis of which is given by $\{\emptyset\} \cup\{X\} \bigcup\{U(x): x \in$ $X\}$, where $U(x)=\{a \in X: x \prec a\} \quad(x \in X)$ is said to be the lower order topology associated to $\precsim$. In the same way, the topology $\tau_{\prec}^{u}$ on $X$, a subbasis of which is given by $\{\emptyset\} \cup\{X\} \bigcup\{L(x): x \in X\}$, where $L(x)=\{b \in X: b \prec$ $x\} \quad(x \in X)$, is said to be the upper order topology associated to $\precsim$.

Definition 4.17. Let $(X, \tau)$ be a topological space. The topology $\tau$ is said to be lower preorderable if there exists a total preorder $\precsim$ on $X$ such that $\tau$ coincides with the lower order topology $\tau_{\prec}^{l}$.

Remark 4.18. As in Remark 4.3 above, the lower preorderability of a topology has already been characterized in terms of suitable bases (see Theorem 3.1 (i) in [24]).

Problem 4.19. Again, topological spaces $(X, \tau)$ such that the topology $\tau$ is the lower order topology of some total preorder on $X$ have already been characterized in [24]. Also, nothing similar is known for topologies induced by other different kinds of binary relations. Thus, given an asymmetric binary relation $\mathcal{R}$ on a nonempty set $X$, we can also consider the sets of the form $L(x)=\{b \in X: b \mathcal{R} x\}(x \in X)$, and consequently define a topology $\tau_{\mathcal{R}}^{l}$ on $X$, a subbasis of which is given by $\{\emptyset\} \cup\{X\} \bigcup\{L(y): y \in X\}$. Here it is an open problem to characterize topologies that coincide with the topology $\tau_{\prec}^{l}$ induced by an interval order or a semiorder $\prec$ on $X$.

Furthermore, the semicontinuous representability property (SRP) has been characterized as follows:

Theorem 4.20. Let $(X, \tau)$ be a topological space. The topology $\tau$ satisfies the semicontinuous representability property if and only if every topology $\tau^{\prime}$ on $X$ such that $\tau^{\prime}$ is coarser than $\tau$ and lower preorderable, satisfies the second countability axiom. 
Proof. See Theorem 5.1 in [24].

Problem 4.21. Here again, we could say that a topological space $(X, \tau)$ satisfies the semicontinuous representability property for interval orders (respectively, semicontinuous representability property for semiorders) (SRP-I.O) (respectively (SRP-S) if for every interval order $\prec$ (respectively, for every semiorder) defined on $X$ and such that all the sets $L(x)=\{b \in X: b \mathcal{R} x\}(x \in X)$ are $\tau$-open, there exists a semicontinuous representation as an interval order (respectively, as a semiorder, in the sense of Scott and Suppes). The study and characterization of the topological spaces $(X, \tau)$ that accomplish SRP-I.O or SRP-S is still an open question. Some pioneering ideas in this direction were launched in [16].

Lemma 4.22. Let $X$ be a nonempty set. Let $\precsim$ denote a total preorder defined on $X$. Then the order topology $\tau_{\prec}$ is second countable if and only if the lower order topology $\tau_{\prec}^{l}$ is second countable.

Proof. See Theorem 4.2 in [24].

Theorem 4.23. The semicontinuous representability property (SRP) implies the continuous representability property (CRP).

Proof. Let $(X, \tau)$ be a topological space. Suppose that $\tau$ satisfies SRP. Take a subtopology of $\tau$ that coincides with the order topology $\tau_{\prec}$ of some total preorder $\precsim$ defined on $X$. By Theorem 4.20, the lower topology $\tau_{\prec}^{l}$ is second countable. Therefore, by Lemma $4.22, \tau_{\prec}$ is second countable. Hence $\tau$ satisfies the continuous representability property (CRP) by Theorem 4.5 .

Remark 4.24. The converse of Theorem 4.23 is not true in general: Following Corollary 4.2 in [23], to see that the converse may fail, consider the first uncountable ordinal $\omega_{1}$. Endowed with the lower order topology $\omega_{1}$ trivially satisfies the continuous representability property (CRP). But is not second countable, so that by Theorem 4.20 it does not accomplishes the semicontinuous representability property (SRP). See $[15,22]$ for further details.

Problem 4.25. Another appealing problem is to identify the semicontinuous representability property (SRP) among the classical ones, or its variants, in General Topology. In this direction, in Corollary 4.6 and Theorem 4.8 in [23] it has been proved that every second countable topological space $(X, \tau)$ satisfies SRP. Also, any topological space that satisfies the semicontinuous representability property SRP is hereditarily separable and hereditarily Lindelöf. The converses are not true. (See also [67] for further details). Thus, SRP can be viewed as a property that falls in the area of separability-countability. It is an open question to identify it exactly with some known property of that kind, or, alternatively, to prove that it is indeed a new intermediate variant.

Theorem 4.26. The semicontinuous representability property is hereditary. That is, given a topological space $(X, \tau)$ that satisfies SRP, and a nonempty subset $Y \subseteq X$, the topological space $\left(Y, \tau_{Y}\right)$ also satisfies $S R P$. (Here $\tau_{Y}$ stands for the topology that $\tau$ induces on $Y)$. 
Proof. See Corollary 5.2 in [22].

Problem 4.27. Since the semicontinuous representability property (SRP) is hereditary, and it implies the continuous representability property (CRP), it immediately follows that SRP carries the hereditary continuous representability property HCRP. It is an open question to study the converse implication. Does HCRP imply SRP or not?

\subsection{Yi's extension property in topological spaces}

A classical characterization of normality in topological spaces is the Tietze extension theorem, so that in a normal topological space a continuous real valued map defined on a closed subset has a continuous extension to the whole space.

At this stage, given a topological space $(X, \tau)$ we may observe that any continuous real-valued map $f: X \rightarrow \mathbb{R}$ immediately defines a continuous total preorder $\precsim$ on $X$ by declaring that $x \precsim y \Longleftrightarrow f(x) \leq f(y) \quad(x, y \in X)$.

This obvious fact suggests to analyze a generalization of the Tietze's extension property. Consider the following key definition (see [22, 82]).

Definition 4.28. A topological space $(X, \tau)$ is said to have Yi's extension property provided that any total preorder defined on a closed subset $A$, and continuous with respect to the relative topology $\tau_{A}$ that $\tau$ induces on the subset $A$, admits a $\tau$-continuous extension to the entire set $X$.

Remark 4.29. This notion was introduced in an approach of utility theory, concerning the problem of extending total preorders (preferences) from a subset of a topological space to the entire space. It was launched by the Korean mathematical economist Gyoseob Yi.

Definition 4.30. Let $(X, \tau)$ be a topological space. $(X, \tau)$ is said to be normal if for each pair of disjoint $\tau$-closed subsets $A, B \subseteq X$ there exists a pair of disjoint $\tau$-open subsets $A^{*}, B^{*} \subseteq X$ such that $A \subseteq A^{*}, B \subseteq B^{*}$. (For basic topological definitions see e.g. $[50,52])$.

It is well-known that this property of being normal is equivalent to an extension property for continuous real-valued functions. This is the "Tietze's extension theorem" (see e.g [81], 15.8).

Theorem 4.31. (Tietze's extension theorem) Let $(X, \tau)$ be a topological space. Then $(X, \tau)$ is normal if and only if for every $\tau$-closed subset $A \subseteq X$, each continuous map $f: A \rightarrow \mathbb{R}$ admits a continuous extension $F: X \rightarrow \mathbb{R}$. Moreover, if $f(X) \subseteq[-a, a]$ for some $a>0$, then $F$ can be chosen so that $F(X) \subseteq[-a, a]$. (This topological property is known as the Tietze's extension property).

Now suppose that $(X, \tau)$ is a normal topological space. An immediate corollary of Tietze's extension theorem states that continuous and representable preferences defined on closed subsets of $X$ can be continuously extended to the entire set $X$. 
Corollary 4.32. Let $(X, \tau)$ be a normal topological space. Let $S \subseteq X$ be a $\tau$-closed subset of $X$. Let $\precsim_{S}$ be a continuous total preorder defined on $S$. Then if $\precsim_{S}$ is representable through a continuous utility function $u_{S}: S \rightarrow \mathbb{R}$, it can also be extended to a continuous total preorder $\precsim X$ defined on the whole $X$.

Proof. Just observe that, by Tietze's theorem, the utility function $u_{S}$ admits a continuous extension to a map $u_{X}: X \rightarrow \mathbb{R}$. Then define $\precsim_{X}$ on $X$ as $x \precsim X y \Longleftrightarrow u_{X}(x) \leq u_{X}(y)(x, y \in X)$.

It can be seen that Yi's extension property implies Tietze's extension property, but the converse does not hold, in general. Therefore, this new extension property of topological spaces, based on the consideration of total preorders defined on its closed subsets, is actually a more restrictive variant of the normality property. Thus, Yi's extension property was initially understood as an strengthtening of Tietze's extension property, in a direction in which we are not interested in extending utility functions, but only total preorders (usually known as preferences in applied contexts arising, e.g., in Mathematical Economics). Observe that Yi's and Tietze's extension properties are not equivalent in the general case. This is because preferences could fail to be representable.

Let us see that, indeed, Yi's extension property is stronger than Tietze's extension property, as claimed before. First we introduce a helpful definition.

Definition 4.33. A topological space $(X, \tau)$ is said to be separably connected if for every $a, b \in X$ there exists a connected and separable subset $C_{a, b} \subseteq X$ such that $a, b \in C_{a, b}$.

Theorem 4.34. Let $(X, \tau)$ be a topological space that satisfies Yi's extension property. Then $(X, \tau)$ is normal. The converse is not true, in general.

Proof. Let $A, B \subseteq X$ be two (nonempty) $\tau$-closed disjoint subsets of $X$. Let $S=A \cup B . S$ is obviously $\tau$-closed. Consider the total preorder $\precsim_{S}$ defined on $S$ as $a_{1} \sim a_{2}$ for every $a_{1}, a_{2} \in A, b_{1} \sim b_{2}$ for every $b_{1}, b_{2} \in B$ and $a \prec b$ for every $a \in A, b \in B$. It is plain that the total preorder $\precsim_{S}$ is continuous on $S$. Applying Yi's extension property, there exists a continuous total preorder $\precsim_{X}$ defined on the whole set $X$, and extending $\precsim_{S}$. We distinguish two possible situations:

1. In the first case, we assume that there exists some element $c \in X \backslash S$ such that $a \prec_{X} c \prec_{X} b$ for every $a \in A, b \in B$. We observe that $B \subseteq U(c)=$ $\left\{x \in X: c \prec_{X} x\right\}$ and also $A \subseteq L(c)=\left\{x \in X: x \prec_{X} c\right\}$. Since $\precsim_{X}$ is $\tau$-continuous, the sets $L(c), U(c)$ are $\tau$-open. In addition, they are disjoint by its own definition.

2. Suppose that there is no element $c \in X \backslash S$ such that $a \prec_{X} c \prec_{X} b$ for every $a \in A, b \in B$. In this case, if we fix an element $\alpha \in A$ and also an element $\beta \in B$, by definition of $\precsim_{S}$ we immediately observe that $A \subseteq L(\beta)=\{x \in$ $\left.X: x \prec_{X} \beta\right\}$ and in the same way, $B \subseteq U(\alpha)=\left\{x \in X: \alpha \prec_{X} x\right\}$. Since $\precsim X$ is $\tau$-continuous, the sets $L(\beta), U(\alpha)$ are $\tau$-open. In addition, they are disjoint because $\alpha \prec_{S} \beta$ by hypothesis. 
Thus we see that $X$ is a normal topological space. But normality is a property that is equivalent to Tietze's extension property.

To see that the converse is not true in general, we should have at hand a counterexample. In [37] (see also [22]) it was proved that on separably connected metric spaces Yi's extension property is equivalent to separability. On the other hand, metric spaces are always normal. Thus, an example of a separably connected metric space that is not separable would fit our purposes. This is easy: consider a non-separable Banach space (e.g., $\ell_{2}(\mathbb{R})$ ) endowed with its norm topology.

Problem 4.35. As far as we know, Yi's extension property has not been identified yet as some of the classical notions related to normality encountered in General Topology. The general problem of characterizing those topological spaces that satisfy Yi's extension property for preferences has not been solved yet.

Under several topological restrictions, Yi's extension property also has strong connections with the continuous representability property (CRP) analyzed before. This was studied in [22]. In fact, both properties may be equivalent in some particular cases, as next theorem states.

Theorem 4.36. On separably connected metric spaces Yi's extension property is equivalent to the continuous representability property CRP.

Proof. See Theorem 3.3 in [22].

Remark 4.37. In general topological spaces, Yi's extension property and the continuous representability property (see Example 4.5 in [22]).

Problem 4.38. Is there some other set of topological conditions on a topological space $(X, \tau)$ such that under those conditions the Yi's extension property amounts to the continuous representability property CRP?

Finally, suppose that we analyze the analogous of Yi's extension property for the semicontinuous case. We would introduce accordingly the following definition.

Definition 4.39. A topological space $(X, \tau)$ is said to have the semicontinuous $Y i$ 's extension property whenever any total preorder defined on a closed subset $A$ and lower semicontinuous with respect to the relative topology $\tau_{A}$ that $\tau$ induces on the subset $A$, admits a $\tau$ - lower semicontinuous extension to the entire set $X$.

At this stage, perhaps surprisingly, it can be proved that any topological space satisfies the semicontinuous Yi's extension property.

Theorem 4.40. In any topological space semicontinuous extensions of total preorders always exist, even without asking the subsets considered to be closed.

Proof. See Theorem 5.1 in [22]. 
Problem 4.41. In spite of the Yi's extension property falling in the area of separation axioms in General Topology, and, in particular, around normality, it is also true that under other topological restrictions Yi's extension property may also fall in the area of separabity-countability. Thus, as aforesaid, in [37] it has been proved that on separably connected metric spaces Yi's extension property amounts to separability. It could be interesting to look for other different families of topological spaces having the same property.

\section{Algebraic representability of different kinds of orderings}

\subsection{Algebraic utility}

Given a nonempty set $X$ endowed with an ordering of a certain kind, it is common that the underlying set $X$ also has some additional algebraic structure. Just to put an example and fix ideas, consider the Euclidean plane $X=\mathbb{R}^{2}$ endowed with the lexicographic order $\precsim_{L}$ given by $(a, b) \precsim_{L}(c, d) \Leftrightarrow(a<$ $c) \vee[(a=c) \wedge(b \leq d)](a, b, c, d \in \mathbb{R})$, and a binary operation $\overline{+}$ defined coordinatewise as $\left(x_{1}, x_{2}\right) \overline{+}\left(y_{1}, y_{2}\right)=\left(x_{1}+y_{1}, x_{2}+y_{2}\right)$, where $\left(x_{1}, x_{2}\right),\left(y_{1}, y_{2}\right) \in \mathbb{R}^{2}$, and + denotes the usual addition of real numbers. Provided that the algebraic operation is compatible with the given ordering, it is then natural to look for numerical representations that not only preserve the ordering, but, in addition, they are also algebraic homomorphism. This is what we would call algebraic utility.

As a matter of fact, these kind of contexts were also analyzed in several papers that, having at hand algebraic structures as the main topic of study, considered some additional compatible ordering so that the existence of orderpreserving homomorphism was a key question to be analyzed (see e.g. [5, 18, $34,59,63])$.

In this framework, the algebraic structure and the ordering should feature some sort of compatibility. A suitable starting point here could be the theory of linearly ordered groups, or, more generally, that of totally ordered semigroups, already analyzed in the classical book [59]. Following, [18], we include here some ideas in this direction, for the sake of completeness.

Definition 5.1. A semigroup $(S, \circ)$ is a nonempty set $S$ equipped with a binary operation o that is associative. If, in addition, $S$ has a null element $e$ such that $s \circ e=e \circ s=s$ holds for every $s \in S$, then $(S, \circ)$ is said to be a monoid. Furthermore, if each element $s$ of a monoid $(S, \circ)$ has an inverse $\bar{s}$ such that $s \circ \bar{s}=\bar{s} \circ s=e$, then $(S, \circ)$ is said to be a group. A semigroup $(S, \circ)$ endowed with a total order $\precsim$ is said to be a totally ordered semigroup if there is a compatibility between the total order $\precsim$ and the binary operation $\circ$ such that $s \precsim t \Leftrightarrow s \circ u \precsim t \circ u \Leftrightarrow u \circ s \precsim u \circ t$ holds for every $s, t, u \in S^{2}$.

\footnotetext{
${ }^{2}$ This property is also known as the translation-invariance of the total order $\precsim$ as regards the binary operation $\circ$. Notice that, in particular, a totally ordered semigroup is always
} 
Given a totally ordered $\operatorname{semigroup}^{3}(S, \circ, \precsim)$ an element $s \in S$ is said to be positive (respectively, negative) if $s \prec s+s$ (respectively, if $s+s \prec s$ ). The set of positive (respectively, negative) elements of $S$ is called the positive cone (respectively, negative cone), and denoted by $S^{+}$(respectively, $S^{-}$). Notice in addition that $S$ may contain an element $e$ that is neither positive nor negative. If this happens, $e$ must be, a fortiori, the null element of $(S, \circ)$ and, in particular, this structure is a monoid. A totally ordered semigroup is said to be positive (respectively, negative) if it only has positive (respectively, negative) elements.

Finally, a totally ordered semigroup $(S, \circ, \precsim)$ is called additively representable if there exists a real-valued function ${ }^{4} f: S \rightarrow \mathbb{R}$ accomplishing that $f(s \circ t)=$ $f(s)+f(t)$ and all $s \precsim t \Leftrightarrow f(s) \leq f(t)$ hold for every $s, t \in S$.

In order to characterize the totally ordered semigroups that are additively representable, we still need to introduce the following definition.

Definition 5.2. A positive semigroup $(S, \circ, \precsim)$ is said to be Archimedean if for every $s, t \in S$ with $s \prec t$, there exists a natural number $n \in \mathbb{N}$ such that $t \prec s^{n}=s \circ \ldots(n-t i m e s) \ldots \circ s$. Moreover, it is called super-Archimedean if for every $s, t \in S$ such that $s \prec t$ there exists a natural number $n \in \mathbb{N}$ such that $s^{n+1} \prec t^{n}$.

In the case of a totally ordered group, a classical result coming from Algebra, and stated by Hölder early in 1901, characterizes the additive representability in terms of Archimedeaness, as follows. (See [63] or else [8], p. 300).

Proposition 5.3. A totally ordered group $(G, \circ, \precsim)$ is additively representable if and only if its positive cone is Archimedean.

In the case of a totally ordered semigroup, the additive representability was characterized by Alimov in 1950, in terms of the super-Archimedean property, as follows (see $[5,45]$ or else the reference-note 21 on p. 26 in [76]).

Proposition 5.4. A totally ordered semigroup $(S, \circ, \precsim)$ is additively representable if and only if its positive cone $S^{+}$is super-Archimedean and its negative

cancellative, namely $s \circ u=t \circ u \Leftrightarrow s=t \Leftrightarrow u \circ s=u \circ t \quad(s, t, u \in S)$.

${ }^{3}$ Despite we are working with totally ordered semigroups, it can be proved that we could actually be working with a totally preordered semigroup, where $\precsim$ is a total preorder but not necessarily a linear order (i.e.: the binary relation $\precsim$ could fail to be antisymmetric). When this happens, we might pass to be working with a quotient space $S / \sim$ whose elements are the indifference classes of the elements of $S$ with respect to $\sim$. That is, given $s \in S$, its corresponding class is the set $\{t \in S: t \sim s\}$. Provided that there is a compatibility between the total preorder $\precsim$ and the binary operation $\circ$ such that $s \precsim t \Leftrightarrow s \circ u \precsim t \circ u \Leftrightarrow u \circ s \precsim u \circ t$ holds for every $s, t, u \in S$, it is easy to see that $S / \sim$ inherits a structure of totally ordered semigroup by considering in a natural way that the binary operation $\circ$ as well as $\precsim$ directly act. on the indifference classes that $\sim$ induces on $S$.

By this reason, in what follows we will be working with totally ordered semigroups, instead of just totally preordered semigroups, unless otherwise stated.

\footnotetext{
${ }^{4}$ In this setting, a mapping $f$ with these properties is said to be an additive utility function.
} 
cone $S^{-}$is also super-Archimedean when endowed with the transpose order $\precsim^{t}$ given by $s \precsim^{t} u \Leftrightarrow u \precsim s \quad(s, u \in S)$.

\section{Remark 5.5.}

(i) The Archimedeaness property on positive totally ordered semigroups is not good enough to warrant the existence of an additive representation. An example is the semigroup $S=(0+\infty) \times(0,+\infty)$ of pairs of strictly positive real numbers, endowed with the lexicographic total order and the binary operation $(s, t) \circ(u, v)=(s+u, t+v)$. It is well-known that this ordered set does not admit a numerical representation, even if we disregard additivity (see e.g. [8], pp. 200-201).

(ii) A positive super-Archimedean totally ordered semigroup $(S, \circ, \precsim)$ is, in particular, Archimedean. (For a detailed proof, see e.g., [45]). The converse is not true: indeed $S=(0+\infty) \times(0,+\infty)$ endowed with the lexicographic ordering $\preceq_{L}$ fails to be super-Archimedean since $(3,3) \prec_{L}(3,4)$ but $(3 k, 4 k) \prec_{L}(3 k+3,3 k+3) \quad(k \geq 1 \in \mathbb{N})$.

(iii) If $(G, \circ, \precsim)$ is an Archimedean totally ordered group, its positive cone is Archimedean and also its negative cone is Archimedean with respect to the transpose order. (See e.g., [45] for a proof and further details, related properties and comments).

Here we introduce some open problems in this direction. Needless to say, on most of them they some partial results have already appeared (See e.g. $[32,39,40,42,72])$.

Problem 5.6. Let $X$ be a nonempty set endowed with a total preorder $\precsim$ and some kind of algebraic structure that may depend on one or more binary operations defined on $X$. Indeed some operations could be external. Thus $X$ could be, say, a semigroup, a monoid, a ring, a vector space, an algebra, etc. Depending on the structure considered, which is the most suitable definition of compatibility between the total preorder $\precsim$ and the algebraic structure?

Problem 5.7. Let $X$ be a nonempty set endowed with an interval order or a semiorder $\prec$, as well as some kind of algebraic structure. What should be understood as a suitable definition of compatibility or intertwined relationship between the ordering $\prec$ and the algebraic structure we have on $X$ ab initio?

At this respect, it is important to point out that sometimes we get a surprising result, as next theorem states (see e.g. [18]).

Theorem 5.8. Let $(S, \circ)$ stand for a semigroup. Let $\prec$ be an interval order defined on $S$. Assume that $\prec$ is translation-invariant (i.e.: $s \prec t \Leftrightarrow s+u \prec$ $t+u \Leftrightarrow u+s \prec u+t$ holds for every $s, t, u \in S$ ). Then if the interval order $\prec$ admits a representation through a pair of real-valued functions $(u, v)$ such that, in addition, both $u$ and $v$ are additive (i.e., $s \prec t \Leftrightarrow v(s) \prec u(t) ; \quad u(s \circ t)=$ $u(s)+u(t) ; \quad v(s \circ t)=v(s)+v(t)$ hold for every $s, t \in S)$, then the associated binary relation $\precsim$ (adjoint of $\prec$ ) is a total preorder. In particular, both $\precsim$ and $\sim$ are reflexive binary relations on $S$. 


\subsection{When algebra meets topology: the continuous alge- braic representability property and related items}

At this stage a next step is to consider a topological space $(X, \tau)$ that is also endowed with an ordering (e.g.: a total preorder $\precsim$ ) as well as with some algebraic structure. Needless to say, we will also assume some kind of compatibility between the algebraic structure and the ordering, as well as some relationship between the topology with respect to the ordering (e.g., assuming that $\precsim$ is $\tau$-continuous), and also between the algebraic structure and the topology (e.g.: assuming that any binary operation is continuous as regards the product topology $\tau \times \tau$ on $X^{2}$ and the given topology $\tau$ on $X$ ).

To fix ideas, following [18], for the sake of completeness we include now some results on numerical representability of a semigroup $(S, \circ)$ endowed with a compatible total preorder $\precsim$ and a topology $\tau$.

Remark 5.9. Even being representable by an additive utility function, a totally ordered semigroup could fail to admit a continuous and additive representation. An example is the semigroup $S=[2,3) \bigcup[4,+\infty) \subset \mathbb{R}$ with the usual sum and order of the reals, and also with the relative topology inherited from the usual one in $\mathbb{R}$. It can bee seen (see e.g. $[32,33]$ ) that the crux for the non-existence of an additive and continuous representation is the discontinuity with respect to the order topology, of the sum in $S$.

Definition 5.10. A topological semigroup $(S, \circ, \tau)$ is a semigroup $(S, \circ)$ endowed with a topology $\tau$ that is compatible with the binary operation $\circ$ in the following sense: it makes continuous ${ }^{5}$ the binary operation $\circ: S \times S \rightarrow S$ that maps the pair $(s, t) \in S \times S$ to the element $s \circ t \in S$. A totally ordered semigroup $(S, \circ, \precsim)$ is said to be a topological totally ordered semigroup provided that the binary operation $\circ$ is continuous with respect to the order topology $\tau_{\precsim}$ on $S$ (and its corresponding product topology on $S \times S$ ).

Remark 5.11. Similarly to Definition 5.10, a topological group is defined as a group $(G, \circ)$ endowed with a topology $\tau$ such that the binary operation $\circ$ is continuous, and also the unary operation of taking inverse in $G$, that assigns to each element $s \in G$ its opposite $\bar{s}$ is a also continuous as regards the topology $\tau$ in $G$.

A famous result due to Nyikos and Reichel (see [72]) proves that any totally ordered group is topological with respect the order topology ${ }^{6}$. However, the analogous result for semigroups is no longer valid (the counterexample shown in Remark 5.9 also fits well here).

The following result is perhaps the best achievement in this setting.

\footnotetext{
${ }^{5}$ Here on $S \times S$ we will consider the product topology coming from $\tau$ on $S$.

${ }^{6}$ Notice that this is, so-to-say, a theorem about "automatic continuity". It actually states that on a totally ordered group $(G, \circ, \precsim)$, both the operation $\circ$ and the unary operation of taking an inverse are, a fortiori, continuous as regards the order topology $\tau_{\precsim}$.
} 
Theorem 5.12. Let $(S, \circ, \precsim)$ be a topological totally ordered semigroup. Suppose in addition that $S$ is given a topology $\tau$ that is natural as regards the the linear order $\precsim$. Asume that the positive cone $S^{+}$is super-Archimedean with respect to $\precsim$, whereas the negative cone $S^{-}$is super-Archimedean with respect to the transpose order. Then $S$ is representable by an additive real-valued function $u: S \rightarrow \mathbb{R}$ such that $u(s \circ t)=u(s)+u(t), s \precsim t \Leftrightarrow u(s) \leq u(t)$ holds for every $s, t \in S$, and the function $u$ is continuous with respect to the given topology $\tau$ in $S$ and the usual Euclidean topology in the real line $\mathbb{R}$.

Proof. See Theorem 2 in [32].

Problem 5.13. In spite of a few studies having already appeared in this literature, a deep analysis of the numerical representability of several kinds of compatible orderings defined on more sophisticated algebraic structures on a topological space $(X, \tau)$ should be considered as a general source of open questions.

To conclude this section, we may consider some algebraic version of the continuous representability property.

Remember that in the topological setting the continuous representability property (CRP) appears whenever we look for ordinal representations of total preorders that, in addition, preserve a nice topological property: namely, the continuity. In the algebraic context, in addition to the order and continuity properties for a utility function, we will ask for a new demanding requirement: that of being an algebraic-homomorphism. Of course, this imposes some kind of compatibility among the ordering, topology, and algebraic structure involved.

To fix ideas, we will include here some results already launched in [27, 39]. In that paper, although one could have begun with some much simpler algebraic structure (e.g.: a totally preordered semigroup) on a topological space $(X, \tau)$, the algebraic structures actually considered were much more sophisticated, namely, totally preordered real algebras.

Definition 5.14. A totally preordered real algebra $\left(X, \precsim,+, \mathbb{R}_{\mathbb{R}}, *\right)$ is a real algebra equipped with a total preorder $\precsim$ which is compatible with the operations ,$+ \bullet_{\mathbb{R}}$ and $*$, i.e.:

(i) $x \precsim y$ implies $x+z \precsim y+z$, for all $z \in X$ (translation-invariance).

(ii) $x \precsim y, 0 \leq \lambda$ implies $\lambda \cdot x \precsim \lambda \cdot y$ (homotheticity).

(iii) $x \precsim y, \mathbf{0} \precsim z$ imply $z * x \precsim z * y$ and $x * z \precsim y * z$ (multiplicativeness. 0 denotes the null element with respect to + ).

Next we define the notion of a topological real algebra.

Definition 5.15. A real algebra $(X,+, \cdot \mathbb{R}, *)$ equipped with a topology $\tau$ is said to be a topological real algebra if $(X, \tau,+, \cdot \mathbb{R})$ is a topological vector space and $*$ is a $\tau$-continuous binary operation on $X \times X$. 
Given a topological space $(X, \tau)$ now introduce the concept of continuous representability of total preorders in the algebraic environment, namely the socalled continuous algebraic representability property.

Definition 5.16. Let $(X,+, \cdot \mathbb{R}, *)$ be a real algebra and $\tau$ a topology on $X$. Then $\tau$ satisfies the continuous algebraic representability property (CARP for short) if every $\tau$-continuous and non-zero ${ }^{7}$ total preorder $\precsim$ defined on $X$, for which the structure $(X, \precsim,+, \cdot \mathbb{R}, *)$ becomes a totally preordered real algebra, admits a continuous utility function which is an algebra-homomorphism (shortly, a continuous algebraic utility function).

The following characterization of the continuous algebraic representation property arises.

Theorem 5.17. Let $(X,+, \cdot \mathbb{R}, *)$ be a real algebra and $\tau$ a topology on $X$. The the following assertions are equivalent:

(i) $\tau$ has $C A R P$

(ii) $\left(X, \tau_{\precsim},+, \cdot \mathbb{R}, *\right)$ is a topological real algebra, for every non-zero and continuous total preorder $\precsim$ that makes $(X, \precsim,+, \cdot \mathbb{R}, *)$ to be a totally preordered real algebra.

Proof. See Theorem 5.12 in [27].

If $\tau$ makes $(X,+, \cdot \mathbb{R}, *)$ to be a topological real algebra then we have the following interesting consequence.

Corollary 5.18. Let $(X,+, \cdot \mathbb{R}, *)$ be a real algebra. Then any topology $\tau$ on $X$ that makes $(X, \tau,+, \cdot \mathbb{R}, *)$ to be a topological real algebra accomplishes the continuous algebraic representability property CARP.

Remark 5.19. In particular, Corollary 5.18 applies to the $n$-dimensional Euclidean space $\mathbb{R}^{n}$ endowed with the usual binary operations defined componentwise. Moreover, in this case it can be easily seen that the continuous algebraic utility functions are of the form $\psi\left(x_{1}, \ldots, x_{j}, \ldots, x_{n}\right)=x_{j}$, for some $j \in\{1, \ldots, n\}$. In other words, any continuous total preorder defined on $\mathbb{R}^{n}$ which is translation-invariant, homothetic and multiplicative is projective.

Problem 5.20. This new approach of continuous algebraic representability properties generates many open questions, such as the following ones:

(i) Explore and analyze continuous algebraic representability properties for total preorders on a topological space $(X, \tau)$ endowed with different kinds of compatible algebraic structures, as, e.g., semigroups, monoids, groups, rings, vector spaces or cones.

\footnotetext{
${ }^{7}$ A total preorder $\precsim$ on $(X,+, \cdot \mathbb{R}, *)$ is said to be non-zero provided that there are $\bar{x}, \bar{y} \in X$ such that $\mathbf{0} \prec \bar{x} * \bar{y}$.
} 
(ii) Explore and analyze continuous algebraic representability properties on a topological space $(X, \tau)$ equipped with some algebraic structure and a compatible interval order and semiorder.

(iii) Define and study concepts relative to semicontinuous algebraic representability properties on a topological space $(X, \tau)$ endowed with some algebraic structure and a compatible ordering of a certain type (e.g.: total preorder, interval order or semiorder).

\section{Alternative versions of utility representations}

\subsection{Representations in codomains different from the real line}

Following [36], we may realize that the main emphasis in the utility theory literature, which deals with the study of order-preserving numerical representations of binary relations that model preferences, different kinds of orderings, etc., has been put on the use of real-valued functions. It is implicitly accepted that the almost exclusive use of the real line $\mathbb{R}$ as the codomain on which the orderpreserving representations (also known as utility functions) take their values is based on the fact that, at least intuitively, utilities are (real) numbers. In addition, it is, perhaps, also implicitly accepted that real-valued utility functions are used because $\mathbb{R}$ is much simpler than other possible codomains, and consequently it is simpler to use numerical quantitative scales rather than qualitative ones to compare elements on a set, or to formalize the preferences of an individual.

Nevertheless, as clearly stated by Herden and Mehta in [61] (see also [21]), we may say that "it is highly desirable and even imperative to begin the development of a theory of the existence and continuity of non-real-valued utility functions". Notice that the real line $\mathbb{R}$, endowed with is usual linear order $\leq$ is just one of the possible linearly ordered sets that we could choose as suitable codomain to represent orderings. And, even being the most popular one, we should not restrict ourselves to use only $\mathbb{R}$. Moreover, as analyzed in [6] there exist different classes of linear orders that fail to admit a utility representation. Of course, those linear orders (e.g., the long line or the lexicographic plane) could also be considered someway as alternative codomains to represent some ordered structures. By cardinality reasons, it is clear that there is no "supreme codomain" $(X, \preceq)$ where $X$ is a nonempty set and $\preceq$ is a linear order on $X$, such that given any nonempty set $Y$ endowed with a total preorder $\precsim$, there exists a map $f: Y \longrightarrow X$ such that $a \precsim b \Leftrightarrow f(a) \preceq f(b)$ holds for every $a, b \in X$. To prove this claim notice that, for any set $X$, the power set $2^{X}$, endowed with a well ordering can never be represented into $(X, \preceq)$ because its cardinality is bigger, so that there is no room in $X$ for a surjective function from $2^{X}$ into $X$ to exist. However, if we restrict the cardinality of the totally preordered set $(Y, \precsim)$ we can still find some powerful result in this direction, such as the classical one obtained by J.S. Chipman in [44]. 
Theorem 6.1. A total preorder $\precsim$ defined on a nonempty set $X$ can always be represented in the classical sense in a codomain of the type $Y=\{0,1\}^{\alpha}$ where $\alpha$ denotes a large enough cardinal number, and $Y$ is endowed with the corresponding lexicographic ordering.

Problem 6.2. Has Theorem 6.1 an analogous result valid for representability of interval orders and semiorders in some way?

Problem 6.3. For a given cardinality $\alpha$, find all the possible codomains $(X, \preceq)$ where $X$ is a nonempty set and $\preceq$ is a linear order on $X$, such that given any nonempty set $Y$ whose cardinality is not bigger than $\alpha$, and assuming that the given set $X$ is endowed with a total preorder $\precsim$, there exists a map $f: Y \longrightarrow X$ such that $a \precsim b \Leftrightarrow f(a) \preceq f(b)$ holds for every $a, b \in X$.

Problem 6.4. For a given cardinality $\alpha$, is there a suitable codomain where any interval order or semiorder defined on a nonempty set $Y$ whose cardinality is smaller than or equal to $\alpha$ can be represented in a feasible way?

We may also study here which are the most suitable codomains to represent total preorders $\precsim$ that are $\tau$-continuous with respect to some topology defined on a nonempty set $X$, and satisfying some additional properties. In this direction, a good example is furnished by the following theorem, whose proof appears in [22].

Theorem 6.5. Every $\tau$-continuous total preorder $\precsim$ on a separably connected topological space $(X, \tau)$ admits an isotonic representation on the double long line endowed with its natural order. Moreover, the representation can be chosen to be continuous with respect to the topology $\tau$ on $X$ and the order topology on the double long line.

Proof. See Lemma 4.1 in [22] for the definition and properties of the double long line, as well as for the proof of this result on representability.

Problem 6.6. Given a topological space $(X, \tau)$ that satisfies some fixed additional properties (e.g, separable connectedness as in Theorem 6.5 above), find some suitable linearly ordered codomain on which any $\tau$-continuous total preorder $\precsim$ can be continuously represented.

Another complementary idea that leads to a change of codomain was analyzed in $[14,21,36,54]$. The key is to consider representations that use only one map, instead of two (as in the case of interval orders), or one function plus a threshold (as in the case of semiorders).

Definition 6.7. Let $\mathcal{C}$ stand for a particular class of binary relations (e.g.: interval orders) that have been defined on a nonempty set $X$. Consider also an standard fixed particular procedure $\mathcal{P}$ to represent the binary relations that belong to the class $\mathcal{C}$ (e.g.: the representability of interval orders through a pair of real-valued functions). We say that a nonempty set $Y$ endowed with a suitable binary relation $\mathcal{R}_{Y}$ is a universal codomain for the class $\mathcal{C}$ with respect 
to the procedure $\mathcal{P}$, if the representability of an element of $\mathcal{C}$ in the sense of the procedure $\mathcal{P}$ is equivalent to the existence of a single map $F: X \rightarrow Y$ such that $x \mathcal{R} y \Leftrightarrow F(x) \mathcal{R}_{Y} F(y)$ holds for every $x, y \in X$.

The key here is to look for representations that use a single map. The main underlying problem is the following (see [14]):

Problem 6.8. Given a particular class $\mathcal{C}$ of binary relations and a fixed procedure $\mathcal{P}$ of representation, find a universal codomain (if any) to represent the elements of that class.

Partial answers to this general problem have already been introduced in this literature for the case of total preorders, interval orders and semiorders (see $[14,21,36,54])$.

\subsection{Utility through functional equations}

Let us go back to the classical representability of a total preorder $\precsim$, defined on a nonempty set $X$, through a real-valued utility function $u: X \rightarrow \mathbb{R}$ such that $x \precsim y \Leftrightarrow u(x) \leq u(y)$ holds for every $x, y \in X$. If we consider the bivariate map $F: X \times X \rightarrow \mathbb{R}$ given by $F(x, y)=u(y)-u(x) \quad(x, y \in X)$ we immediately observe that $x \precsim y \Leftrightarrow F(x, y) \geq 0$ holds. In addition, the function $F$ satisfies the so-called Sincov's functional equation (see [78]), namely $F(x, y)+F(y, z)=F(x, z) \quad(x, y, z \in X)$.

Following [29], we introduce the definition of some classical functional equations in two variables.

Let $X$ be a nonempty set. Let $F: X \times X \rightarrow \mathbb{R}$ be a real-valued bivariate map defined on $X$.

Definition 6.9. The bivariate map $F$ is said to satisfy:

(i) The Sincov functional equation if $F(x, y)+F(y, z)=F(x, z)$ holds for every $x, y, z \in X$,

(ii) the Sincov functional equation (second version) if $F(x, y)+F(y, z)+$ $F(z, x)=0$ holds for every $x, y, z \in X$,

(ii) the separability functional equation if $F(x, y)+F(y, z)=F(x, z)+F(y, y)$ holds for every $x, y, z \in X$,

(iv) the restricted separability functional equation if $F(x, y)+F(y, z)=F(x, z)+$ $F(t, t)$ holds for every $x, y, z, t \in X$,

(v) the Ferrers functional equation if $F(x, y)+F(z, t)=F(x, t)+F(z, y)$ holds for every $x, y, z, t \in X$,

(vi) the semitransitivity functional equation if $F(x, y)+F(y, z)=F(x, t)+$ $F(t, z)$ holds for every $x, y, z, t \in X$. 
Proposition 6.10. Let $X$ be a nonempty set. Let $F: X \times F \rightarrow \mathbb{R}$ be a bivariate map. The following statements hold:

(i)) Both versions of Sincov functional equation are equivalent.

(ii) The separability equation is equivalent to the Ferrers equation.

(iii) The restricted separability equation is equivalent to the semitransitivity equation.

Proof. See Proposition 1 in [29].

Let us see now how those functional equations may intervene in alternative representations of total preorders, interval orders and semiorders.

Theorem 6.11. Let $X$ be a nonempty set. Let $\precsim$ be a total preorder on $X$. Then the following statements are equivalent:

(i) The total preorder $\precsim$ is representable by means of a utility function $u$ : $X \rightarrow \mathbb{R}$ such that $x \precsim y \Leftrightarrow u(x) \leq u(y) \quad(x, y \in X)$.

(ii) There exists a real-valued bivariate map $F: X \times X \rightarrow \mathbb{R}$ that satisties the Sincov functional equation and, in addition, $x \prec y \Leftrightarrow F(x, y)>0$ holds for every $x, y \in X$.

Proof. See Proposition 5 in [29].

Let us see now what happens as regards interval orders.

Theorem 6.12. Let $X$ be a nonempty set. Let $\prec$ be an interval order on $X$. Then the following statements are equivalent:

(i) The interval order $\prec$ is representable by means of a pair of real-valued functions $u, v: X \rightarrow \mathbb{R}$ such that $x \prec y \Leftrightarrow v(x)<u(y) \quad(x, y \in X)$.

(ii) There exists a real-valued bivariate map $F: X \times X \rightarrow \mathbb{R}$ that satisfies the separability functional equation and, in addition, $x \prec y \Leftrightarrow F(x, y)>0$ holds for every $x, y \in X$.

Proof. See Proposition 6 in [29].

Finally, with respect to semiorders, we also get an analogous result, that goes as follows.

Theorem 6.13. Let $X$ be a nonempty set. Let $\prec$ be a semiorder on $X$. Then the following statements are equivalent:

(i) The semiorder $\prec$ is representable in the sense of Scott and Suppes by means of a real-valued function $u: X \rightarrow \mathbb{R}$ such that $x \prec y \Leftrightarrow u(x)+1<$ $u(y) \quad(x, y \in X)$. 
(ii) There exists a real-valued bivariate map $F: X \times X \rightarrow \mathbb{R}$ that satisfies the semitransitivity functional equation as well as $F(x, x)=-1$ for every $x \in X$, and, in addition, $x \prec y \Leftrightarrow F(x, y)>0$ holds for every $x, y \in X$.

Proof. See Proposition 7 in [29].

Problem 6.14. Given a nonempty set $X$ and a bivariate map $F: X \times X \rightarrow \mathbb{R}$ we may associate to $F$ a binary relation $\mathcal{R}_{F}$ on $X$ given by $x \mathcal{R}_{F} y \Leftrightarrow F(x, y)>$ $0 \quad(x, y>0)$ (see [29]). Suppose now that $F$ satisfies some functional equation. What can be said about the corresponding binary relation $\mathcal{R}_{F}$ ? Does it correspond to some classical kind of ordering (e.g.: a total preorder, an interval order or a semiorder)?

\subsection{Utility theory in the fuzzy setting}

Following [2], we pay now attention to the possibility of defining comparisons or preferences that are fuzzy instead of crisp. Suppose that we consider different kinds of orderings on a nonempty set $X$. Our idea is to establish some sort of comparison or qualitative scale between its elements. Mathematically, when this approach is formalized a variety of classical binary relations (e.g. total preorders, interval orders, etc.) naturally arise. Usually, when dealing with those different relations, if we consider that an element $x \in X$ is related to another element $y \in X$, it happens that the relationship is either void $(=0)$ or total $(=1)$ : That is, either the elements are not related at all, or they are fully related. No intermediate situation is allowed. This is the crisp setting, and it is the most usual one in classical Utility Theory.

However, a common feature, that nowadays, in more modern studies, is arising in many models is the consideration of comparisons or suitable binary relations that are graded. For instance, this is done in order to describe an intensity in the relationship between two given elements. Or this setting can also appear in situations of uncertainty. In these cases, two elements could be related at any level between 0 and 1 . Of course, now the binary relation becomes fuzzy.

Definition 6.15. Given a nonempty set $U$, called universe, a fuzzy subset $X$ of $U$ is a map $\mu_{X}: U \rightarrow[0,1] .^{8}$ Also, a fuzzy binary relation defined on a nonempty universe $U$ is a function $\mathcal{F}: U \times U \rightarrow[0,1]$. In other words $\mathcal{F}$ becomes a fuzzy subset of the Cartesian product $U \times U$.

The classical properties of (crisp) binary relations should then be generalized to the fuzzy setting in some suitable way. To put an example, one may say that a fuzzy relation $\mathcal{F}$ on a universe $U$ is reflexive if $F(t, t)=1$ holds for every $t \in U$. However, definitions such as asymmetry, transitivity, etc., as well as certain operations such as intersections, unions, complements, etc., depend on the choice of a suitable triangular norm (see [2]) for details). In fact, there

\footnotetext{
${ }^{8}$ It is usual that the map $\mu_{X}$ and the corresponding fuzzy set $X$ are used interchangeably if this does not give rise to confusion.
} 
are equivalent definitions of such concepts in the crisp setting (working with classical sets, that is, non-fuzzy), that, when extended to the fuzzy approach, are no longer equivalent, and give rise to many possible different theories and approaches, depending on the definitions considered in each fuzzy context.

Problem 6.16. Extend to the fuzzy setting in a suitable way concepts such as reflexivity, asymmetry, transitivity, negation, intersection and union, as well as classical orderings arising in the crisp setting, namely total preorders, interval orders and semiorders.

Remark 6.17. In [47, 65] several different kinds of fuzzy semiorders were introduced and analyzed. In those studies it is put in evidence that many nonequivalent definitions of the concept of a fuzzy semiorder are possible.

When working with fuzzy binary relation, we would also ask ourselves about what could be a suitable way to represent them numerically, that is, the main general question is the following.

Problem 6.18. Implement a suitable way to convert fuzzy binary relations of certain types into numerical (crisp) scales.

A possible way to do so, is to consider the $\alpha$-cuts of a fuzzy binary relation. Any of this $\alpha$-cuts is a crisp (i.e.: non-fuzzy) binary relation. So, if we are able to represent those $\alpha$-cuts in some suitable way, we could use those numerical representations as a global or full representation of the fuzzy binary relation considered a priori. This has been analyzed in [2] for the case of fuzzy total preorders.

The definition of an $\alpha$-cut goes as follows.

Definition 6.19. Given a fuzzy relation $\mathcal{F}$ on a universe $U$, and a real number $\alpha \in[0,1]$, we define the $\alpha$-cut of $\mathcal{F}$ as the crisp binary relation on $U$ given by $\mathcal{F}_{\alpha}=\left\{(s, t) \in U^{2}: F(s, t) \geq \alpha\right\}$.

Remark 6.20. Obviously, the consideration of $\alpha$-cuts could also generate new extensions of the classical concepts of orderings from the crisp to the fuzzy setting. To put an example, we could adopt the following definition of a fuzzy total preorder: a fuzzy binary relation $\mathcal{F}$ is said to be a fuzzy total preorder if $\mathcal{F}_{\alpha}$ is a crisp total preorder for any $\alpha \in[0,1]$.

Problem 6.21. Study and analyze the fuzzy binary relations such that all their $\alpha$-cuts are total preorders, interval orders or semiorders. Find suitable kinds of numerical representations for each case.

\section{Conclusion}

Many advances have been achieved in Utility Theory in recent years. In particular, new approaches arose related to numerical representability of certain kinds of qualitative scales, such as total preorders, interval orders and semiorders. 
Therefore, it could be helpful for any researcher in this framework to have at hand not only a clear account of these new achievements, but, also, an exhaustive list of open questions that may lead to new streams and trends in research.

This has been the main aim of the present work. Our intention has been to highlight most of the open problem still encountered in this general theory and its most classical branches.

\section{Acknowledgments}

This work is partially supported by the research projects ECO2015-65031R, MTM2015-63608-P (MINECO/ AEI-FEDER, UE), and TIN2016-77356-P (MINECO/ AEI-FEDER, UE).

We are grateful to two reviewers for their valuation suggestions and comments.

Thanks are also given to the organizers and participants in the congress SUMTOPO 2019, 34th Summer Conference on Topology and its Applications, University of the Witwatersrand, Johannesburg (South Africa) 1-4 July 2019, for their helpful discussions and comments on the contents of our contribution, a substantial part of which was presented there.

\section{References}

[1] F.J. Abrísqueta, J.C. Candeal, R.G. Catalán, J.R. De Miguel, E. Induráin: Generalized Abel functional equations and numerical representability of semiorders, Publ. Math. Debrecen 78 (3-4), 557-568 (2011).

[2] L. Agud, R.G. Catalán, S. Díaz, E. Induráin, S. Montes: Numerical representability of fuzzy total preorders, Internat. J. Comput. Intell. Systems (IJCIS), 5(6), 996-1009 (2012).

[3] J.C.R. Alcantud, M.J. Campión, J.C. Candeal, R.G. Catalán, E. Induráin: On the structure of acyclic binary relations, Springer International Publishing AG, part of Springer Nature 2018 J. Medina et al. (Eds.): IPMU 2018, CCIS 855, pp. 3-15 (2018).

[4] F. Aleskerov, D. Bouyssou, B. Monjardet: Utility maximization, choice and preference (second edition), Springer, Berlin, 2007.

[5] N.G. Alimov: On ordered semigroups (in Russian), Izv. Akad. Nauk SSSR Ser. Mat. 14, 569-576 (1950).

[6] A.F. Beardon, J.C. Candeal, G. Herden, E. Induráin, G.B. Mehta: The non-existence of a utility function and the structure of non-representable preference relations. J. Math. Econom. 37, 17-38 (2002).

[7] A. Beja, I. Gilboa: Numerical representations of imperfectly ordered preferences. A unified geometric exposition, J. Math. Psych. 36, 426-449 (1992). 
[8] G. Birkhoff: Lattice Theory, 3rd ed., American Mathematical Society: Providence RI, U.S.A.,1967.

[9] G. Bosi, M.J. Campión, J.C. Candeal, E. Induráin: Interval-valued representability of qualitative data: the continuous case, Internat. J. Uncertain. Fuzziness Knowledge-Based Systems 15 (3), 299-319 (2007).

[10] G. Bosi, M.J. Campión, J.C. Candeal, E. Induráin, M.E. Zuanon: Isotonies on ordered cones through the concept of a decreasing scale, Math. Social Sci. 54, 115-127 (2007).

[11] G. Bosi, J.C. Candeal, E. Induráin: Continuous representability of interval orders and biorders, J. Math. Psych. 51, 122-125 (2007).

[12] G. Bosi, J.C. Candeal, E. Induráin, E. Olóriz, M. Zudaire: Numerical representations of interval orders, Order 18, 171-190 (2001).

[13] G. Bosi, A. Estevan, J. Gutiérrez García, E. Induráin: Continuous representability of interval orders: the topological compatibility setting, Internat. J. Uncertain. Fuzziness Knowledge-Based Systems 23 (3), 345-365 (2015).

[14] G. Bosi, J. Gutiérrez García, E. Induráin: Unified representability of total preorders and interval orders through a single function: the lattice approach, Order 26, 255-275 (2009).

[15] G. Bosi, G. Herden: On the structure of completely useful topologies, Appl. Gen. Topol. 3 (2), 145-167 (2002).

[16] G. Bosi, M.E. Zuanon: Semicontinuous representability of interval orders on a metrizable topological space, Int. J. Contemp. Math. Sci. 2 (17-20), 853-858 (2007).

[17] D.S. Bridges, G.B. Mehta: Representations of Preference Orderings, Springer Verlag, Berlin, 1995.

[18] M.J. Campión, G. Arzamendi, L.M. Gandía, E. Induráin: Entropy of chemical processes versus numerical representability of orderings, J. Math. Chem. 54, 503-526 (2016).

[19] M.J. Campión, J.C. Candeal, R.G. Catalán, A. Giarlotta, S. Greco, E. Induráin, J. Montero: An axiomatic approach to finite means, Inform. Sci. 457-458, 12-28 (2018).

[20] M.J. Campión, J.C. Candeal, A.S. Granero, E. Induráin: Ordinal representability in Banach spaces, in J.M.F. Castillo, W.B. Johnson (Eds.) Methods in Banach Space Theory, pp. 183-196, Cambridge University Press, UK, 2006.

[21] M.J. Campión, J.C. Candeal, E. Induráin: Representability of binary relations through fuzzy numbers, Fuzzy Sets and Systems 157 1-19 (2006). 
[22] M.J. Campión, J.C. Candeal, E. Induráin: On Yi's extension property for totally ordered topological spaces, J. Korean Math. Soc. 43 (1), 159-181 (2006).

[23] M.J. Campión, J.C. Candeal, E. Induráin: Semicontinuous orderrepresentability of topological spaces, Bol. Soc. Mat. Mexicana (3) 15, 81-89 (2009).

[24] M.J. Campión, J.C. Candeal, E. Induráin: Preorderable topologies and order-representability of topological spaces, Topology Appl. 159, 2971-2978 (2009).

[25] M.J. Campión, J.C. Candeal, E. Induráin, G.B. Mehta: Order embeddings with irrational codomains: Debreu properties of real subsets, Order 23, 343-357 (2006).

[26] M.J. Campión, J.C. Candeal, E. Induráin, G.B. Mehta: Representable topologies and locally connected spaces, Topology Appl. 154, 2040-2049 (2007).

[27] M.J. Campión, J.C. Candeal, E. Induráin, G.B. Mehta: Continuous order representability properties of topological spaces and algebraic structures, J. Korean Math. Soc. 49 (3), pp. 449-473 (2012).

[28] M.J. Campión, J.C. Candeal, E. Induráin, M. Zudaire: Continuous representability of semiorders, J. Math. Psych. 52, 48-54 (2008).

[29] M.J. Campión, L. De Miguel, R.G. Catalán, E. Induráin, F.J. Abrísqueta: Binary relations coming from solutions of functional equations: orderings and fuzzy subsets, Internat. J. Uncertain. Fuzziness Knowledge-Based Systems 25 Suppl. 1, 19-42 (2017).

[30] M.J. Campión, E. Falcó, J.L. García-Lapresta, E. Induráin: Assigning numerical scores to linguistic expressions, Axioms 6 (3), UNSP 19 (2017).

[31] M.J. Campión, C. Gómez-Polo, E. Induráin, A. Raventós-Pujol: A survey of the mathematical foundations of axiomatic entropy: Representability and orderings, Axioms 7, 29 (2018).

[32] J.C. Candeal, J.R. De Miguel, E. Induráin: Extensive measurement: Continuous additive utility functions on semigroups, J. Math. Psych. 40 (4), 281-286 (1996).

[33] J.C. Candeal, J.R. De Miguel, E. Induráin: Topological additively representable semigroups, J. Math. Anal. Appl. 210 385-389 (1997).

[34] J.C. Candeal, J.R. De Miguel, E. Induráin, G.B. Mehta: Representations of ordered semigroups and the physical concept of entropy, Appl. Gen. Topol. 5 (1), 11-23 (2004). 
[35] J.C. Candeal, A. Estevan, J. Gutiérrez-García, E. Induráin: Semiorders with separability properties, J. Math. Psych. 56, 444-451 (2012).

[36] J.C. Candeal, J. Gutiérrez García, E. Induráin: Universal codomains to represent interval orders, Internat. J. Uncertain. Fuzziness Knowledge-Based Systems 17 (2), 197-219 (2009).

[37] J. C. Candeal, C. Hervés, E. Induráin: Some results on representation and extension of preferences, J. Math. Econom. 29, 75-81 (1998).

[38] J.C. Candeal, E. Induráin: Semiorders and thresholds of utility discrimination: Solving the Scott-Suppes representability problem, J. Math. Psych. 54, 485-490 (2010).

[39] J.C. Candeal, E. Induráin, J.A. Molina: Numerical representability of ordered topological spaces with compatible algebraic structure, Order 29, 131-146 (2012).

[40] J.C. Candeal, E. Induráin, M. Sanchis: Order representability in groups and vector spaces, Expo. Math. 30, 103-123 (2012).

[41] J.C. Candeal, E. Induráin, M. Zudaire: Numerical representability of semiorders, Math. Social Sci. 43 (1), 61-77 (2002).

[42] J.C. Candeal-Haro, E. Induráin Eraso: A note on linear utility, Econom. Theory 6 (3), 519-522 (1995).

[43] J.C. Candeal-Haro, E. Induráin Eraso: Utility representations from the concept of measure, Math. Social Sci. 26(1), 51-62 (1993).

[44] J.S. Chipman: The foundations of utility, Econometrica 28(2) 193-224 (1960).

[45] J.R. De Miguel, J.C. Candeal, E. Induráin: Archimedeaness and additive utility on totally ordered semigroups, Semigroup Forum 52, 335-347 (1996).

[46] G. Debreu: Representation if a preference ordering by a numerical function, in: Thrall, R., Coombs, C., Davies, R. (eds.) Decision Processes, pp. 159 166. Wiley, New York (1954).

[47] S. Díaz, E. Induráin, B. De Baets, S. Montes: Fuzzy semi-orders: the case of t-norms without zero divisors, Fuzzy Sets and Systems 184, 52-67 (2011).

[48] J.P. Doignon, A. Ducamp, J.C. Falmagne: On realizable biorders and the biorder dimension of a relation, J. Math. Psych. 28, 73-109 (1984).

[49] M. Droste: Ordinal scales in the theory of measurement, J. Math. Psych. 31, 60-82 (1987).

[50] J. Dugundji: Topology, Allyn and Bacon, Boston, 1966. 
[51] S. Eilenberg: Ordered topological spaces, Amer. J. Math. 63, 39-45 (1941).

[52] R. Engelking: General topology. Revised and completed edition, Heldermann Verlag, Berlin, 1989.

[53] A. Estevan, J. Gutiérrez García, E. Induráin: Further results on the continuous representability of semiorders, Internat. J. Uncertain. Fuzziness Knowledge-Based Systems 21 (5), 675-694 (2013).

[54] A. Estevan, J. Gutiérrez García, E. Induráin: Numerical representation of semiorders, Order 30, 455-462 (2013).

[55] P.C. Fishburn: Utility Theory for Decision-Making, Wiley, New York, 1970.

[56] P.C. Fishburn: Intransitive indifference with unequal indifference intervals, J. Math. Psych. 7, 144-149 (1970).

[57] P.C. Fishburn: Intransitive indifference in preference theory: a survey, Oper. Res. 18(2), 207-228 (1970).

[58] P.C. Fishburn: Interval Orders and Interval Graphs, Wiley, New York, 1985.

[59] L. Fuchs: Partially Ordered Algebraical Systems, Pergamon Press, Oxford UK, 1963.

[60] G. Herden: Topological spaces for which every continuous total preorder can be represented by a continuous utility function, Math. Social Sci. 22, 123-136 (1991).

[61] G. Herden, G. B. Mehta: The Debreu Gap Lemma and some generalizations, J. Math. Econom. 40(7) 747-769 (2004).

[62] G. Herden, A. Pallack: Useful topologies and separable systems, Appl. Gen. Topol. 1 (1), 61-82 (2000).

[63] O. Hölder: Der Axiome der Quantität und die Lehre vom Mass, Berichte über die Verhandlungen der Königlich Sachsischen Gesellschaft der Wissenschaften zu Leipzig, Math. Phys. Kl. 53, 1-64 (1901).

[64] E. Induráin, V. Knoblauch: On topological spaces whose topology is induced by a binary relation, Quaest. Math. 36 (1), 47-65 (2013).

[65] E. Induráin, D. Martinetti, S. Montes, S. Díaz, F.J. Abrísqueta: On the preservation of semiorders from the fuzzy to the crisp setting, Internat. $J$. Uncertain. Fuzziness Knowledge-Based Systems 19 (6), 899-920 (2011).

[66] R.D. Luce: Semiorders and a theory of utility discrimination, Econometrica 24, 178-191 (1956). 
[67] D.J. Lutzer, H.R. Bennet: Separability, the countable chain condition and the Lindelf property on linearly ordered spaces, Proc. Amer. Math. Soc. 23 (3), 664-667 (1969).

[68] K.L. Manders: On JND representations of semiorders, J. Math. Psych. 24, 224-248 (1981).

[69] G.B. Mehta: A remark on a utility representation theorem of Rader, Economic Theory 9, 367-370 (1997).

[70] B. Monjardet: Axiomatiques et propriétés des quasi-ordres, Math. Sci. Hum. 63, 51-82 (1978).

[71] L. Nachbin: Topology and Order, Van Nostrand Reinhold, New York, 1970.

[72] P.J. Nyikos, H.C. Reichel: Topologically orderable groups, Gen. Topology Appl. 5 (3), 195-204 (1975).

[73] E. Olóriz, J.C. Candeal, E. Induráin: Representability of interval orders, J. Econom. Theory 78 (1), 219-227 (1998).

[74] S. Purisch: A history of results on orderability and suborderability, in: Handbook of the History of General Topology 2, pp. 689-702, Kluwer, Dordrecht, 1998.

[75] T. Rader: The existence of a utility function to represent preferences, Review of Economic Studies 30, 229-232 (1963).

[76] F.S. Roberts, R.D. Luce: Axiomatic thermodynamics and extensive measurement, Synthese 18, 311-326 (1968).

[77] D. Scott, P. Suppes: Foundational aspects of theories of measurement, J. Symbolic Logic 23, 113-128 (1958).

[78] D.M. Sincov: Über eine Funktionalgleichung, Arch. Math. Phys. (3) 6, 216-227 (1903).

[79] A.N. The, A. Tsoukias: Numerical representation of PQI interval orders, Discrete Appl. Math. 147 (1), 125-146 (2005).

[80] J. Van Dalen, E. Wattel: A topological characterization of ordered spaces, Gen. Topol. Appl. 3, 347-354 (1973).

[81] S. Willard: General Topology, Reading, Massachussets U.S.A.,1970.

[82] G. Yi: Continuous extension of preferences, J. Math. Econom. 22, 547-555 (1993). 